Textures and Microstructures, 1989, Vol. 10, pp. 265-307

Reprints available directly from the publisher

Photocopying permitted by license only

(C) 1989 Gordon and Breach Science Publishers Inc.

Printed in the United Kingdom

\title{
Advantages of Neutron Diffraction in Texture Analysis
}

\author{
H. J. BUNGE
}

Department of Physical Metallurgy, Technical University of Clausthal, FRG

(Received 3 February, 1989)

Neutron diffraction texture analysis is based on pole figure measurement followed by pole figure inversion. Neutron diffraction pole figure measurement is quite similar to that by X-rays. There are, however, several advantages in detail which are mainly due to the lower absorption coefficient. Besides these quantitative differences, there is one principle difference between the two methods. Neutron diffraction allows the magnetic texture to be measured which is not possible by X-rays. The paper gives a survey on the advantages of neutron diffraction texture analysis which are only counteracted by the limited availability and higher costs of neutron diffraction.

KEY WORDS: Pole figure measurement, low absorption coefficient, position sensitive detector, time-of-flight-method, magnetic texture analysis.

\section{INTRODUCTION}

The texture of a polycrystaline material is defined as the orientation distribution function of its crystallites

$$
f(g)=\frac{d V / V}{d g} ; \quad \begin{aligned}
g & =\left\{\left\{\varphi_{1}, \phi, \varphi_{2}\right\}\right\} \\
d g & =\frac{\sin \varphi}{8 \pi^{2}} d \varphi_{1}, d \phi, d \varphi_{2}
\end{aligned}
$$

Thereby $d V / V$ is the volume fraction of crystallites having a crystallographic orientation $g$ within the limits $d g$. The orientation $g$ of the crystallographic axes with respect to a sample-fixed coordinate system may be described by the Euler angles $\varphi_{1}, \phi, \varphi_{2}$, or by many other orientation parameters, see e.g. Figure 1. 

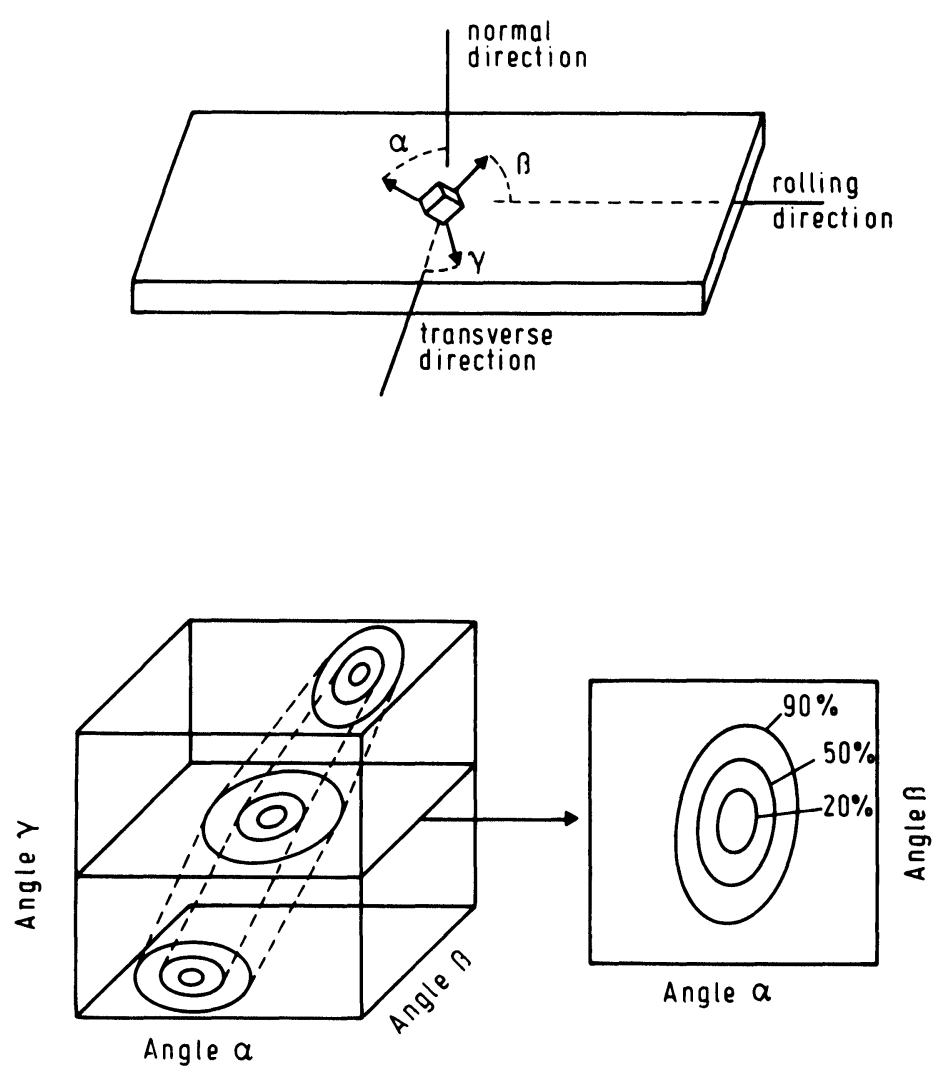

Figure 1 The texture is the orientation distribution function of the crystallites. It depends on three orientation parameters.

Texture measurements can be carried out with the help of different kinds of radiation using either "imaging" or diffraction methods. These radiations are mainly visible light, X-rays, neutrons, and electrons.

Imaging methods may be based on light and electrons whereas diffraction methods may be applied with $\mathrm{X}$-rays, neutrons and electrons as is shown in Table 1.

The methods of texture analysis can also be classified according to whether they are individual crystallite methods or polycrystal methods. 
Table 1 Texture measurement with different radiations, using imaging and diffraction methods

\begin{tabular}{lll}
\hline Radiation & \multicolumn{2}{c}{ Texture measurement by } \\
& imaging methods & diffraction methods \\
\hline Light & $\times$ & $\times$ \\
X-rays & & $\times$ \\
Neutrons & $\times$ & $\times$ \\
Electrons & $\times$ & \\
\hline
\end{tabular}

1) If lateral and vertical resolving power is higher than grain size, then individual orientation measurements can be used. Thereby the crystallographic orientation of a crystallite may be obtained from a) crystallographically oriented features of the crystallite seen in its image in "direct space" (imaging method) and b) the diffraction pattern ("image in reciprocal space") (diffraction method).

2) If the lateral resolving power of the method is much smaller than grain size then "collective diffraction patterns" (pole figures) can be used to obtain information about the orientation distribution.

In the first group of methods, the (discontinuous) individual orientations have to be converted somehow into a statistically relevant continuous orientation distribution function, Figure 2.

In the second method, statistically relevant continuous distribution functions are directly obtained, however, not the complete distribution function depending on all three orientation parameters. The obtained pole figures are only two-dimensional "projections" taken along certain projection paths in the orientation space from which the three-dimensional distribution function has to be constructed by mathematical methods which are comparable to the well-known "computer tomography." This is shown schematically in Figure 3 (see e.g. Bunge, 1969, 1982, 1987).

In the first group of methods statistical relevance can only be improved by increasing the number of orientation measurements i.e. by increasing the experimental effort (considerably).

In the pole figure method statistical relevance can be increased (nearly unlimited) by increasing the size of the sample, compared to grain size, provided the whole sample volume can be made to 

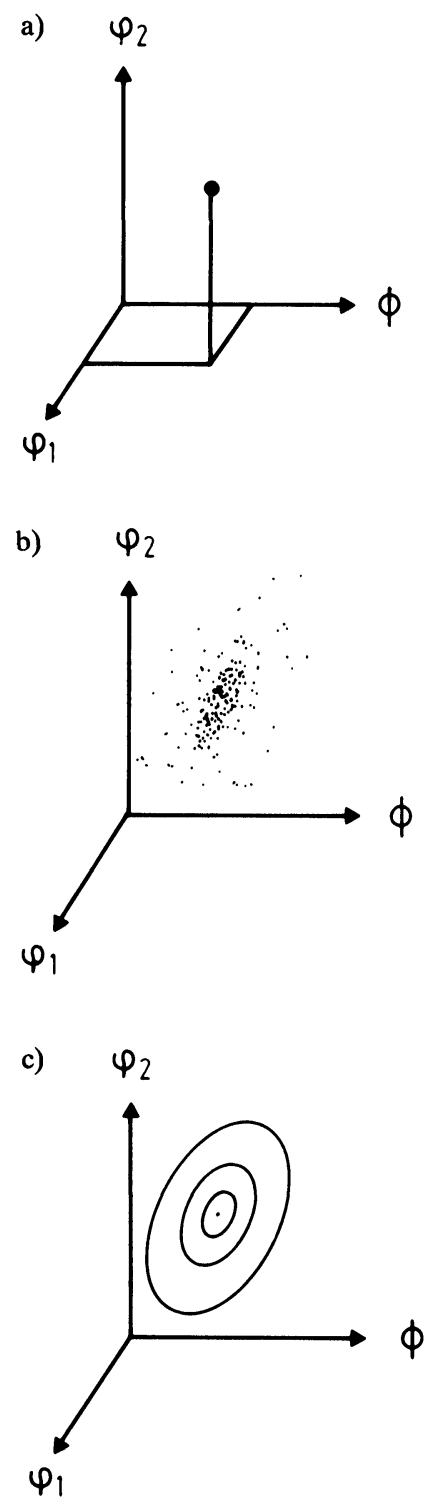

Figure 2 Texture determination from individual orientation measurements. a) The orientation of an individual grain represented in Euler space. b) A "cloud" of orientation points. c) The continuous orientation density distribution. 

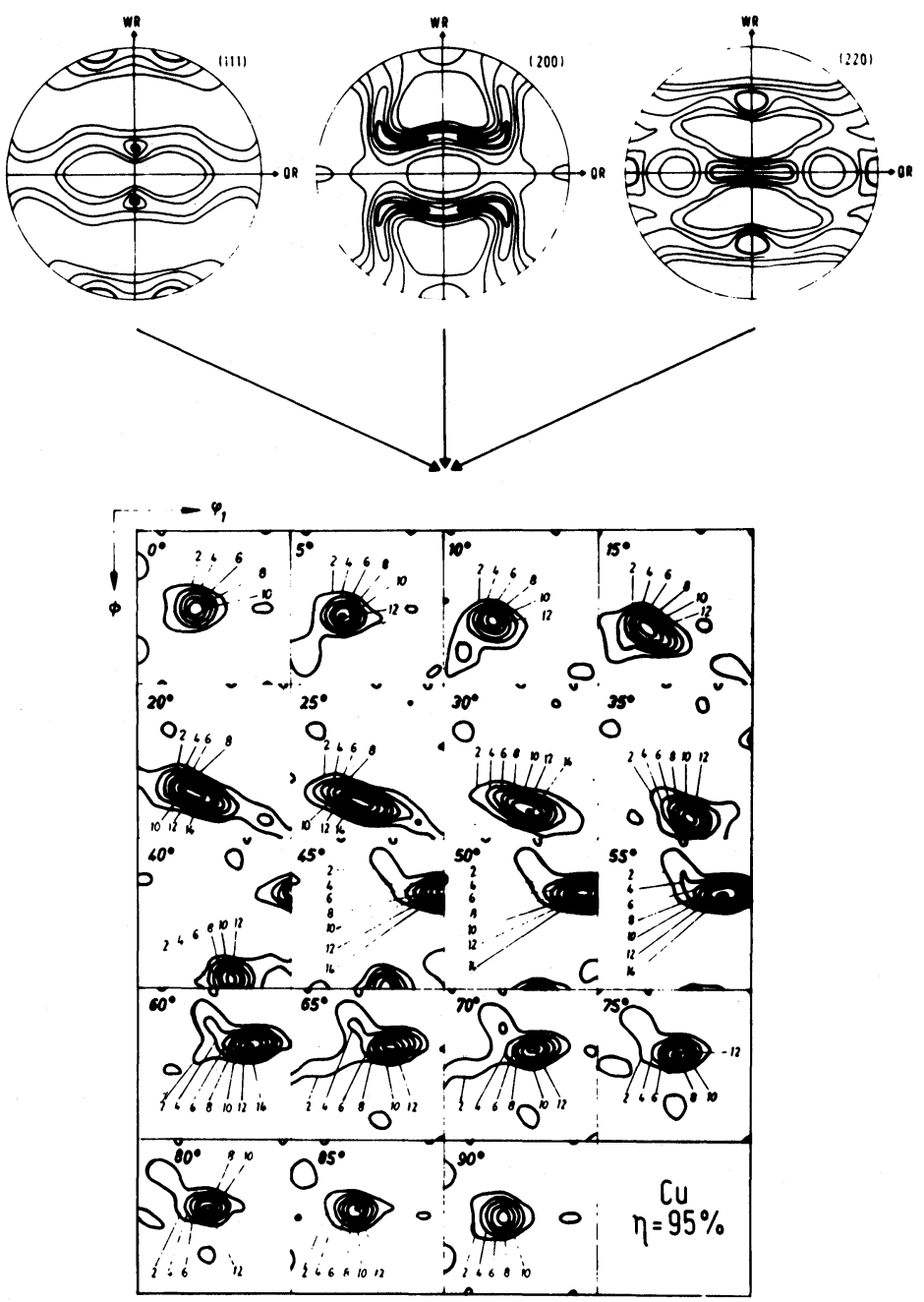

Figure 3 Texture determination from pole figures.

contribute to the measurement at the same time. Hence, with the pole figure method statistically relevant results can be obtained in reasonable time. The virtual disadvantage of this method, the necessity of a mathematical inversion processs is no longer an essential obstacle since highly performant computer programs for pole figure inversion are now available. 
Furthermore, pole figure measurement can be nearly completely automatized by using computer-operated texture goniometers (e.g. Puch, Klein, Bunge, 1984). The individual orientation measurements, on the other hand, have only been "semiautomatized" up to now. Hence, the great majority of texture determinations have been carried out by pole figure measurement followed by pole figure inversion.

As was already mentioned, pole figure measurement can, in principle, be carried out by three kinds of radiation i.e. by X-rays, neutrons, and electrons. It is the purpose of the present paper to provide an overview over the advantages and disadvantages of neutron-diffraction as a means of texture analysis.

\section{POLE FIGURE MEASUREMENT}

A pole figure is defined as the orientation distribution function of an individual crystal direction $h$ perpendicular to a reflecting lattice plane $(h k l)$

$$
P_{h}(y)=\frac{d V / V}{d y}: \quad \begin{aligned}
y & =\{\alpha, \beta\} \\
d y & =\sin \alpha d \alpha d \beta
\end{aligned}
$$

Thereby $d V / V$ is the volume fraction of crystals the direction $h$ of which is parallel to the sample direction $y$. In the conventional method of pole figure measurement one incident and one reflected beam are being used, the bisectrix of which defines the diffraction vector $s$. Pole figure measurement then requires to bring all sample directions $y$-one after the other-into the diffraction direction $s$ and each time to measure the diffracted intensity. This method requires a diffractometer in order to fix the Bragg-angle $\vartheta$ and a sample rotation device which is usually a Eulerian cradle shown schematically in Figure $4 \mathrm{~b}$. The Eulerian cradle allows to rotate the sample through three angles which are usually denoted by $\omega \chi \phi$ as is also shown in Figure $4 \mathrm{~b}$. For pole figure measurement, the sample has to be rotated through two angles $(\alpha \beta)$. Hence, different scanning procedures may be applied e.g. by using only the angles $\chi$ and $\phi$ or the angles $\omega$ and $\chi$ respectively. (This corresponds to the reflection and transmission method in X-ray diffraction although the principle distinction between these two methods does not apply to 
a)

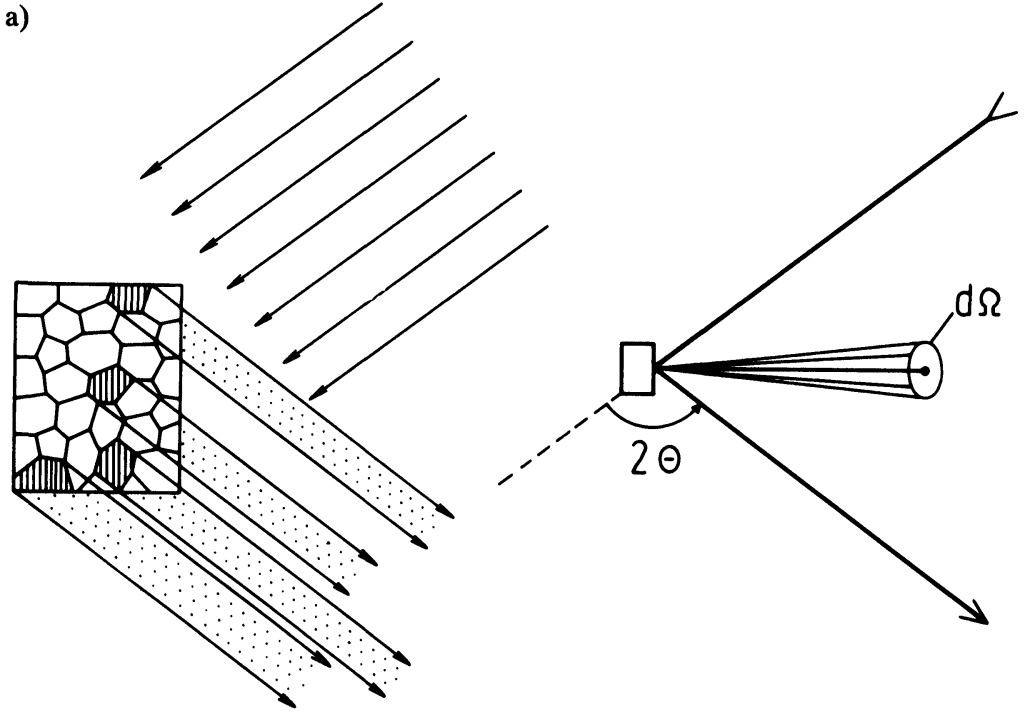

b)

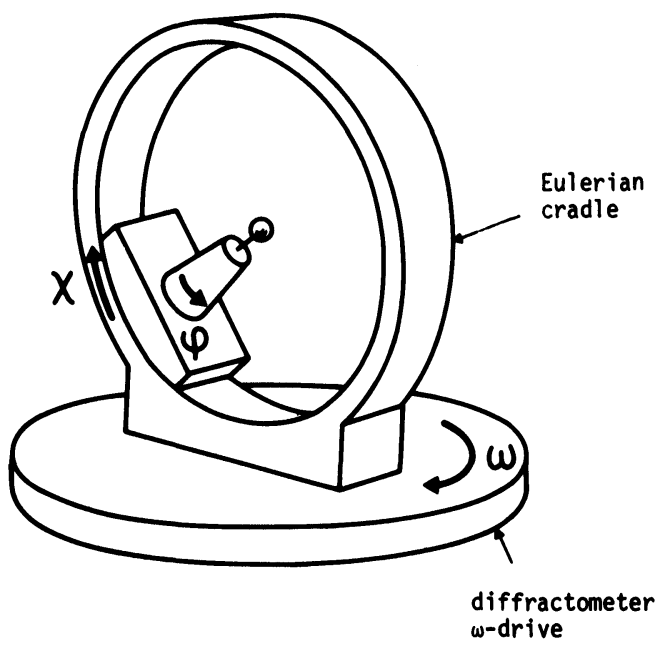

Figure 4 Principles of pole figure measurement using a Eulerian cradle. a) Diffraction of an incident beam in a polycrystalline sample. b) The Eulerian cradle. 
neutron diffraction.) In the conventional method of pole figure measurement the whole angular range $0 \leq \alpha \leq 90^{\circ}, 0 \leq \beta \leq 360^{\circ}$ is to be scanned in steps of $\Delta \alpha, \Delta \beta$ (which may be constant in the whole range or not). In each sample position the reflected intensity is to be measured and the result is to be represented in the form of a continuous pole density function as defined in Eq. (2). The whole procedure is then to be repeated with the next pole figure, i.e. with another Bragg-angle. One thus obtains a set of pole figures which provide the input data for the pole figure inversion process shown schematically in Figure 3. The whole measuring procedure can be carried out automatically when a computer operated texture goniometer is available. This method is very similar in X-ray diffraction and neutron diffraction.

It should be mentioned here, that in electron diffraction a different method can be used in which the sample is only rotated through one angle. A second angle is obtained by deflecting incident and/or reflected beam magnetically which is not possible with X-rays and neutrons (see e.g. Schwarzer and Weiland, 1986).

Pole figure measurement with all three types of radiation is normally carried out in the angular dispersive mode. This means that monochromatic radiation is being used and reflection at the various lattice planes (with different lattice plane spacings $d$ ) is distinguished by different Bragg angles according to Bragg's law

$$
n \cdot \lambda=2 d_{(h k l)} \cdot \sin \vartheta_{(h k l)}
$$

In the case of X-rays the spectrum consists of two parts, a continuous (white) spectrum (the Bremsspectrum) and the characteristic line spectrum Figure 5. The peak intensity of the latter one is usually much higher than that of the white spectrum. In most cases of X-ray texture analysis, it is thus sufficient either to work with the spectrum as it is or to use only a $\beta$-filter. A crystal monochromator is then not necessary. The diffraction of the white radiation contributes a small part to the background scattering which is being taken into account by background measurements taken at both sides of the correct Bragg angle. The background is often taken as a function of the pole figure angle $\alpha$ but not as a function of $\beta$. This is certainly not strictly correct but it is often a sufficient compromise.

The spectrum of thermal neutrons does not contain a characteris- 


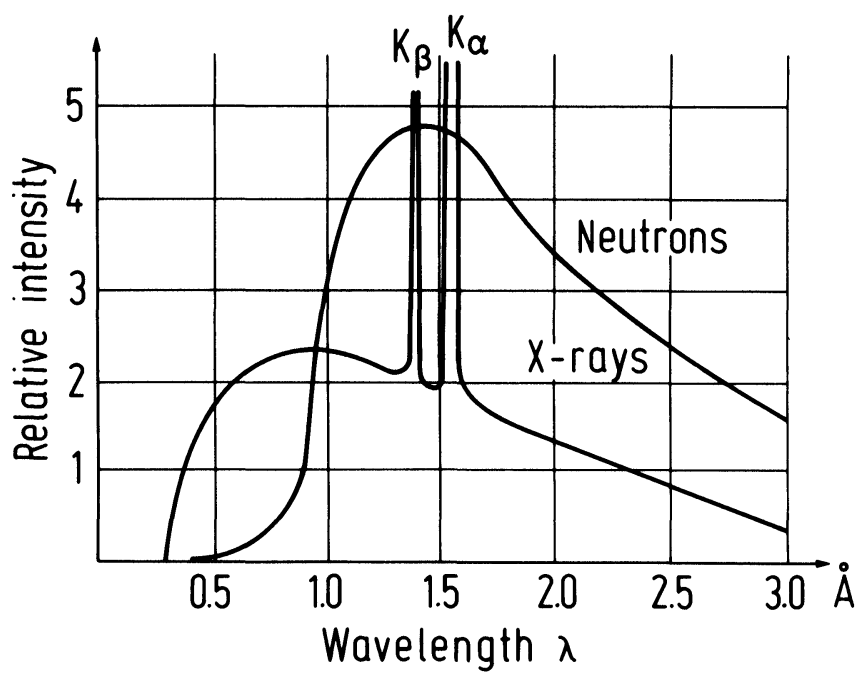

Figure 5 Spectral characteristics of X-rays and neutrons.

tic line spectrum as is seen in Figure 5. Hence, it is then necessary to use a monochromator. Typical monochromator materials are copper, aluminium, lead, silicon, germanium and graphite which select a wave length according to Bragg's law Eq. (3). In order to change the wave length it is thus necessary to change the monochromator or to change the Bragg angle $\vartheta$ or both. In this case the reflected beam (i.e. the incident beam of the texture goniometer) has to pass through the monochromator shielding at different angles and the position of the goniometer itself has to be changed. This requires a higher technical effort than to work with just one "take off angle." Hence, the available wavelengths are often restricted to only a few (notwithstanding the continuous nature of the neutron spectrum).

According to Bragg's law a monochromator may allow a shorter wavelength to pass through (an integer fraction $\lambda / n$ ). For the case of texture analysis this is, however, not a serious problem. It leads only to the superposition of pole figures $(h k l)$ and $(n h, n k, n l)$ which are identical.

The angular divergence of incident and reflected beam of the monochromator determines the spectral width $\Delta \lambda$ of the monochro- 
matized beam and this, in turn, gives rise to a certain line broadening in the measured diffraction spectrum of the sample. On the other hand, the higher the divergencies and the higher $\Delta \lambda$ the higher is the measured intensity and hence, the shorter is the required measuring time. For texture analysis in most cases a rather strong instrumental line broadening can be tolerated (at least if material, such as metals, with not too linerich spectra are being studied). Hence, an optimization of the divergencies is used in texture analysis which may be different from the requirements in other diffraction methods (e.g. high-resolution structure analysis). This must be taken into consideration when multi-purpose diffractometers are being used for texture analysis. These diffractometers are mostly optimized for crystal structure analysis.

\section{TEXTURE ANALYSIS BY NEUTRON DIFFRACTION}

The three kinds of radiation, X-rays, neutrons, and electrons have greatly different penetration depths in matter due to different absorption coefficients. At the same time, also the lateral resolving power in the three methods is different as is shown schematically in Table 2. Accordingly, the irradiated sample volume may be different by nearly twenty orders of magnitude for electron diffraction and neutron diffraction.

Even if one takes into account that in X-ray diffraction texture measurement an additional sample integration may be applied which increases the irradiated sample size without decreasing the

Table 2 Penetration depth, lateral resolving power, and sample volume for the three kinds of radiation

\begin{tabular}{l|l|l|l|}
\hline & Neutrons & X-rays & Electrons \\
\hline $\begin{array}{l}\text { Penetration } \\
\text { depth [mm] }\end{array}$ & 10 & $10^{-1}-10^{-2}$ & $10^{-4}$ \\
$\begin{array}{l}\text { Lateral } \\
\text { resolving } \\
\text { power [mm] }\end{array}$ & 10 & $1-10^{-2}$ & $10^{-3}-10^{-6}$ \\
$\begin{array}{l}\text { Sample } \\
\text { volume } \\
{\left[\mathrm{mm}^{3}\right]}\end{array}$ & $10^{3}$ & $10^{-1}-10^{-6}$ & $10^{-10}-10^{-16}$ \\
\hline
\end{tabular}


angular resolving power (see e.g. Bunge and Puch, 1984) then still a difference of about three orders of magnitude in sample size remains between neutron and X-ray diffraction. This bigger sample size constitutes one of the main advantages of neutron diffraction compared with X-ray diffraction texture analysis.

After neutron diffraction became available with the advent of nuclear reactors after 1945, it was applied to texture measurement for the first time by Brockhouse in 1953. At that time, one of the major advantages of neutron diffraction over X-ray diffraction for texture analysis-the much higher accuracy of the results-could, however, not yet be exploited. In fact, it could not even be proven, since quantitative accuracy figures for texture measurements were not yet available. Hence, the major disadvantage of neutron diffraction, its much higher experimental effort dominated, such that virtually no more texture measurements were carried out by neutron diffraction in the following fifteen years.

A reliability criterion for pole figures based on the series expansion method (Bunge, 1966) showed lateron that neutron diffraction pole figures were much more accurate than those obtained by X-rays (Schläfer, 1968). Furthermore, this higher accuracy was actually needed for the calculation of ODF from pole figures. Hence, Bunge and Tobisch (1968) and Bunge, Tobisch and Sonntag (1971) determined the ODF of cold rolled copper from neutron diffraction pole figures. Textures of $\alpha$-Brass were determined by Bunge and Tobisch (1972) and Bunge, Tobisch and Mücklich (1974) and the rolling and recrystallization textures of low carbon steels were investigated, this way, by Schläfer and Bunge 1974 and Bunge, Schleusener and Schläfer (1974). The methodical details as well as the advantages of neutron diffraction for texture analysis were reviewed by Szpunar (1976) and by Kleinstück $e$ t al. (1976). From this time on, neutron diffraction texture analysis was continuously carried out in several neutron diffraction laboratories including magnetic scattering (Szpunar et al., 1968; Stott and Hutchinson, 1973; Hennig et al., 1981).

Besides metallic materials, also non-metallics were studied (Walter et al. 1981, Brokmeier 1982) and the method proved to be especially valuable for geological studies (Bunge, Wenk, Pannetier 1982, Bankwitz et al. 1984, Höfler, Schäfer, Will 1986, Höfler, Will 1986, Drechsler et al. 1984). Texture development in steels was 
studied by Schreiber et al. (1978) and by Klimanek et al. (1981). The high-speed PSD method was applied to dynamic recrystallization investigations by Jensen et al. (1981) and a detailed review was given by Welch (1986). The time-of-flight method was especially developed by Szpunar et al. (1968), Nosik et al. (1979), Feldmann et al. (1981), Betzl et al. 1984 and a review of this method is contained in the paper by Feldmann in this volume.

\section{LARGE GRAIN SAMPLES}

The statistical relevance of pole figure measurement is limited by the number of grains involved in each pole figure point. This number depends on the angular resolving power and on the total number of grains in the irradiated sample volume. Comparing X-ray and neutron measurements with the same angular resolving power, it is thus possible to obtain the same statistical relevance in neutron diffraction samples having bigger grain size by one order of magnitude in diameter (i.e. three orders in grain volume).

\section{TEXTURE INHOMOGENEITY AND GLOBAL TEXTURES}

The texture of a material is often inhomogeneous, i.e. It is different in samples taken from different places in the material, as is shown schematically in Figure 6a. It is then necessary to distinguish between local textures and the global texture of the whole material. Inhomogeneities may be divided roughly into macro- and microinhomogeneities, examples of which are shown in Figure $6 \mathrm{~b}$ and $c$ respectively. An important macroinhomogenity is, for instance, the variation of the texture of a sheet from surface to the interior, Figure $6 \mathrm{~b}$. A prominent microinhomogeneity occurs in the shear bands, Figure $6 \mathrm{c}$. In Table 3 it is shown how local and global textures in the macro- and microscale can be distinguished by the three kinds of radiation. The global texture on a macroscale is needed for instance when macroscopic properties of the material are being considered. If $E(g)$ is any physical property of a crystallite which depends on its orientation $g$ (e.g. resistance to plastic deformation) then the corresponding macroscopic property is 
a)

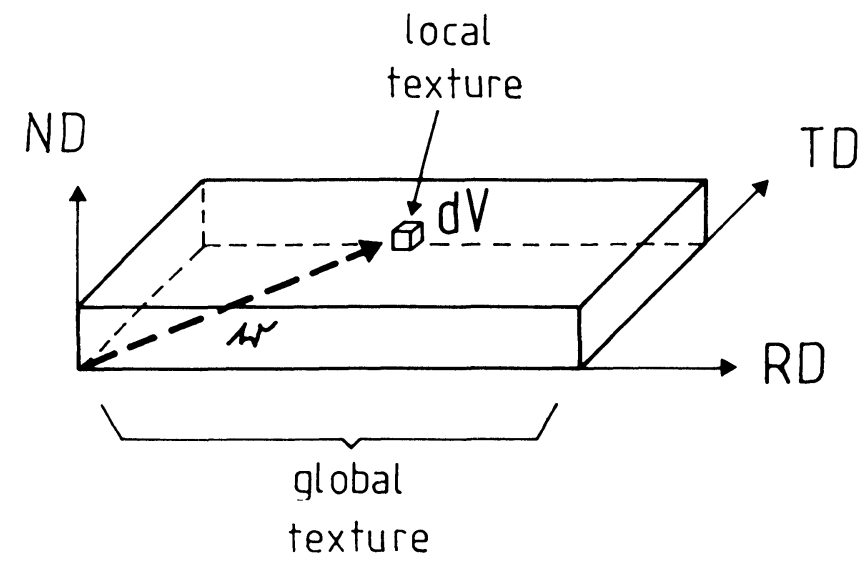

b)

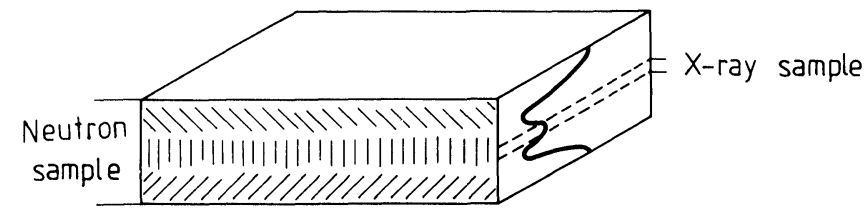

c)
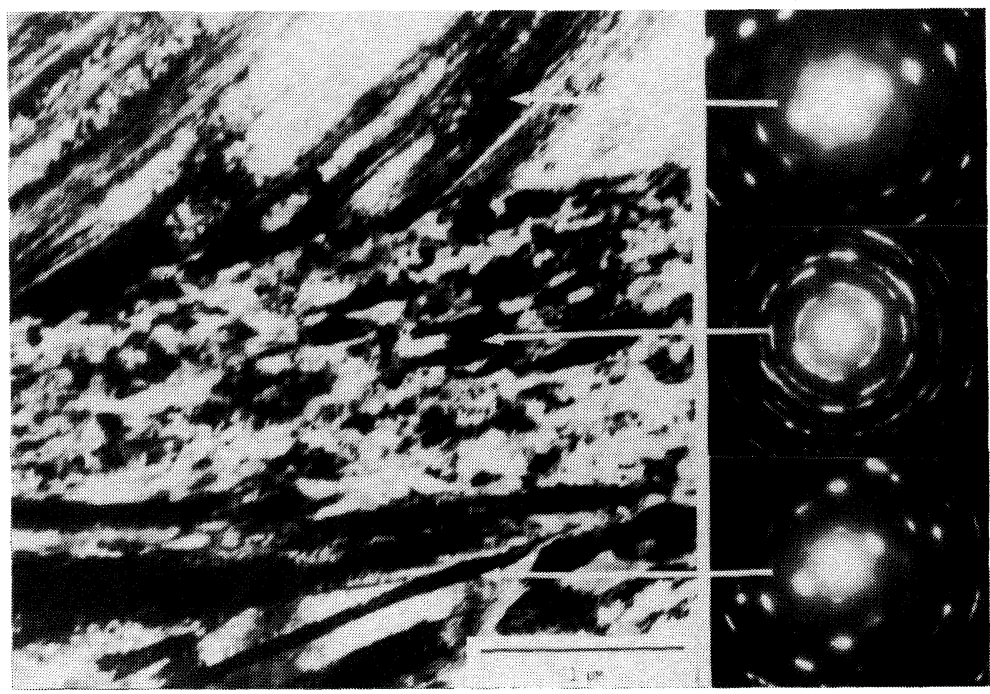

Figure 6 Inhomogenity of textures. a) Definition of local and global texture. b) Macroinhomogeneity. c) Microinhomogeneity (shear band). 
Table 3 Detection of macro- and microinhomogeneities by the three kinds of radiation

\begin{tabular}{llll}
\hline Inhomogeneities & Neutrons & X-rays & Electrons \\
\hline $\begin{array}{llll}\text { macro } \\
\text { micro }\end{array}$ & $\begin{array}{l}\text { global } \\
\text { global }\end{array}$ & $\begin{array}{l}\text { local } \\
\text { global }\end{array}$ & $\begin{array}{l}\text { local } \\
\text { local }\end{array}$ \\
\hline
\end{tabular}

given by

$$
\bar{E}=\oint E(g) \cdot f(g) d g
$$

Thereby $f(g)$ is the orientation distribution of all crystals of the macroscopic sample i.e. the global texture of the material.

In the case of plastic aniostropy as a fundamental property for deep drawing sheet, thicknesses in the range of several milimeters are often to be considered. The plastic properties of such materials are correlated with the global texture obtained by the $x$-ray composite sample method or directly by neutron diffraction. The surface texture measured by the x-ray back-reflection method gives

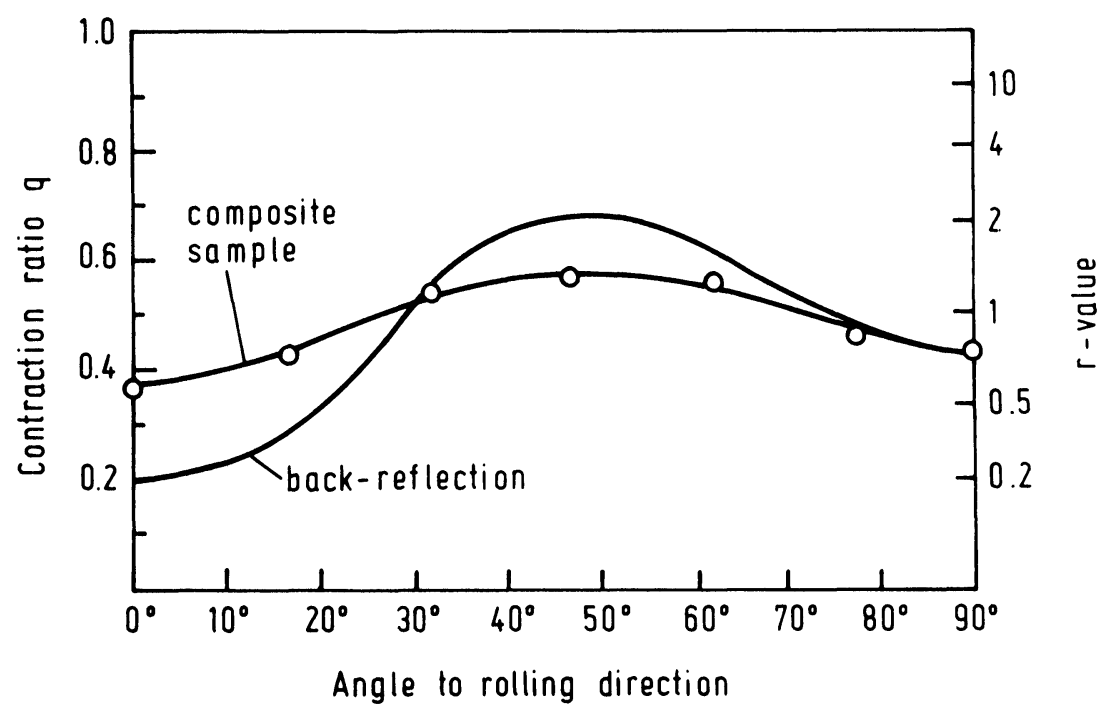

Figure 7 Variation of the $r$-value of plastic anisotropy in the sheet plane of a steel sample measured mechanically (circles) and calculated from texture measurements in the whole volume (composite sample) and the surface (back-reflection). 
quite different results. This is illustrated in Figure 7. Hence, neutron diffraction is especially advantageous for technological samples.

\section{ACCURACY OF THE MEASUREMENT}

Pole figure measurement is based on intensity measurements of the reflected beam. The intensity depends on the volume fraction of crystallites in reflection position (the pole density value which is to be measured) and the absorption of incident and reflected beam in the sample. This has to be taken into account by an absorption correction factor

$$
A=e^{-\mu \cdot x}
$$

where $\mu$ is the linear absorption coefficient, and $x$ is the total path of incident and reflected beam in the sample. The absorption factor $A$ may be calculated and used as a correction factor if the shape of the sample (and hence the path $x$ ) is correctly known. If the shape of the sample is only incorrectly known, then the absorption factor and hence the resulting pole density values are falsified. Differentiating
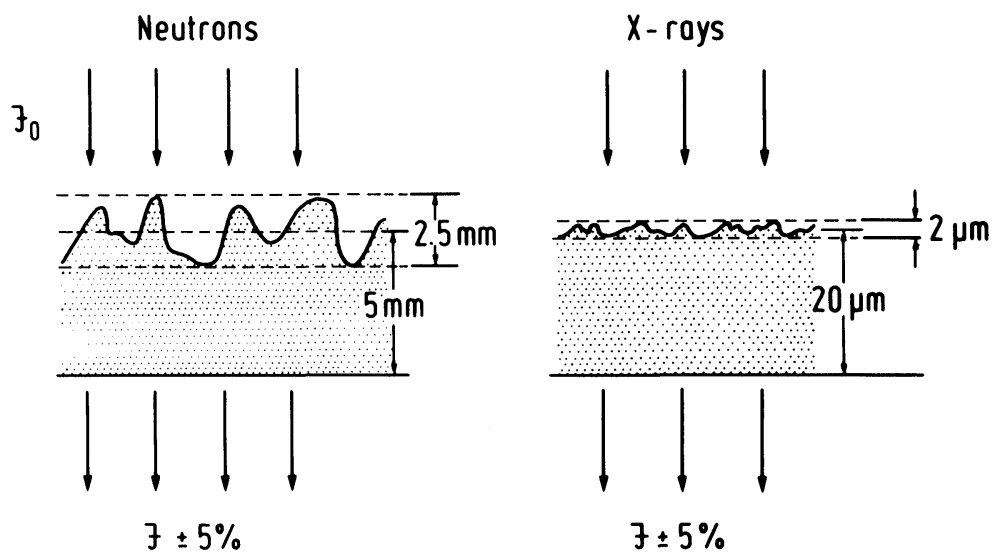

Figure 8 The accuracies $d x / x$ required for a neutron and an X-ray sample in order to obtain a resulting accuracy of $5 \%$ in the correction factor and hence in the pole density values. 
Eq. (5) with respect to $x$ gives

$$
\frac{d A}{A}=-\mu \cdot x \cdot \frac{d x}{x}
$$

Hence, the relative error $d A / A$ of the correction factor depends on

a)

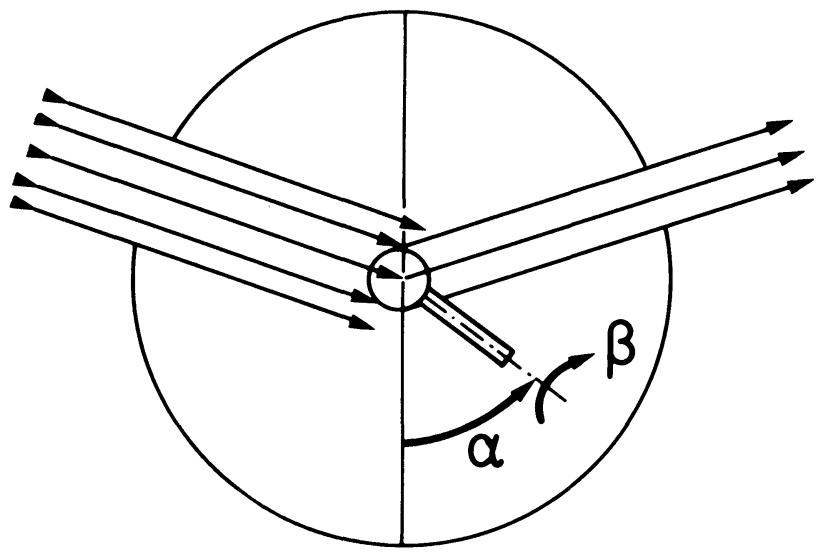

b)

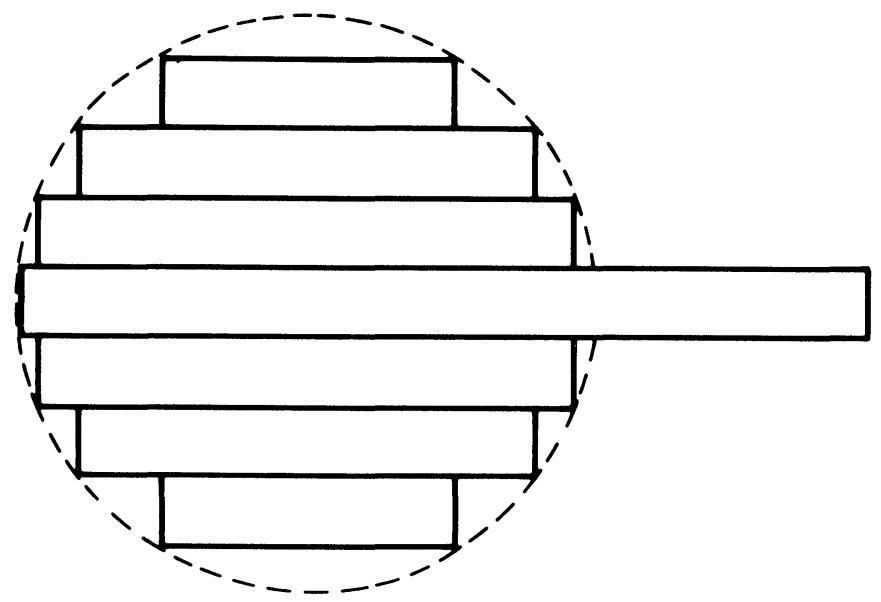

Figure 9 The spherical sample method in neutron diffraction texture analysis. a) The absorption coefficient $A$ is independent of the pole figure angles $\alpha \beta$. b) a "spherical sample" may have an approximate form. 
the relative error $d x / x$ of the sample shape but it also increases with $\mu \cdot x$. In the case of neutron samples usually much lower $\mu \cdot x$ values are being used than in the case of X-rays and also the sample size $x$ is much higher for neutrons than for X-rays. Hence the possible tolerance $d x$ of the sample size is much higher for neutrons than for $\mathrm{X}$-rays. Two typical examples are compared in Figure 8 , where $d A / A=5 \%$ is assumed. Hence, the accuracy of neutron texture measurements can easily be made better by an order of magnitude compared to X-ray measurements.

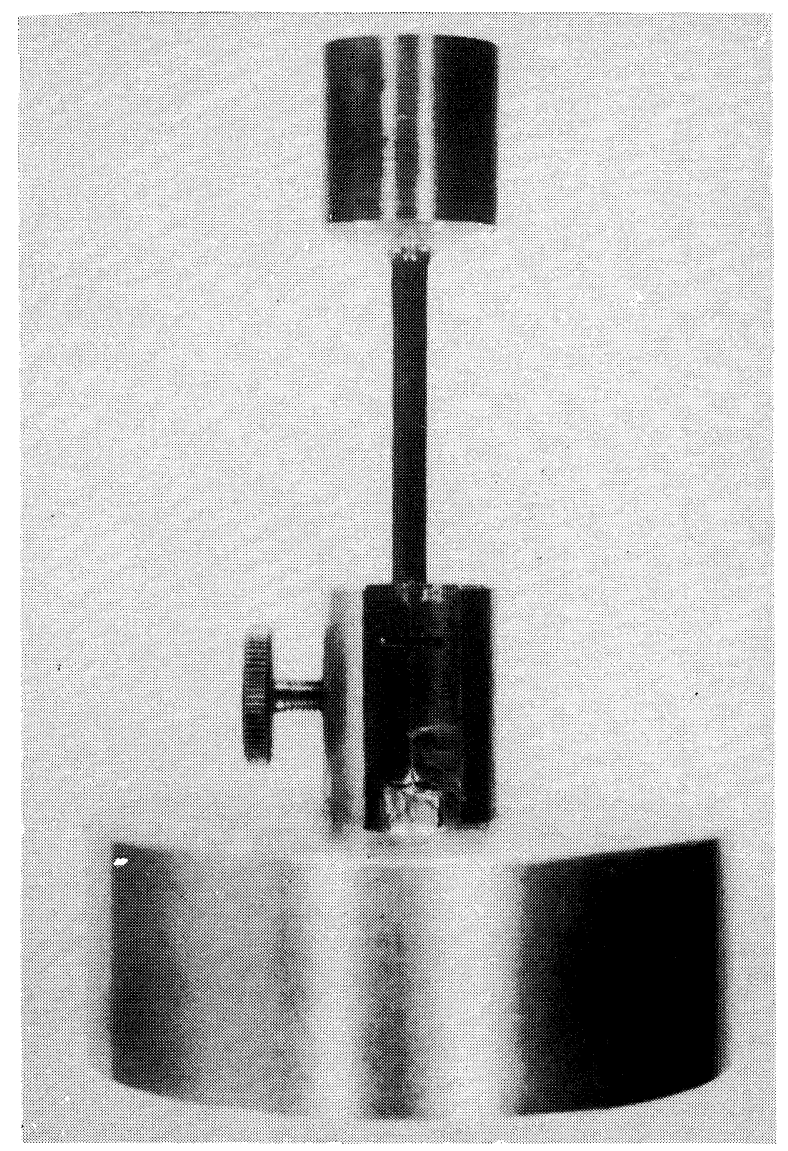

Figure 10 A "spherical" sample for neutron texture analysis. 
The highest accuracy can be obtained when a spherical sample is being used (Tobisch, Bunge 1972). Then the absorption factor $A$ is independent of the pole figure angles $(\alpha, \beta)$ as is shown in Figure 9a. Because of the low $\mu \cdot x$-value in Eq. (6) a "sphere" may have the shape of Figure $9 \mathrm{~b}$ or it may even be a cylinder as in Figure 10.

The resulting accuracy is shown in Figure 11 where error coefficients $\Delta F$ are drawn as a function of the order $\lambda$. It is seen that neutron diffraction with the spherical sample method gives much better results than X-ray diffraction. Especially, the strong increase of these coefficients at low $\lambda$-values does not occur. It has been shown that the increase is due to systematic errors, Figure 12

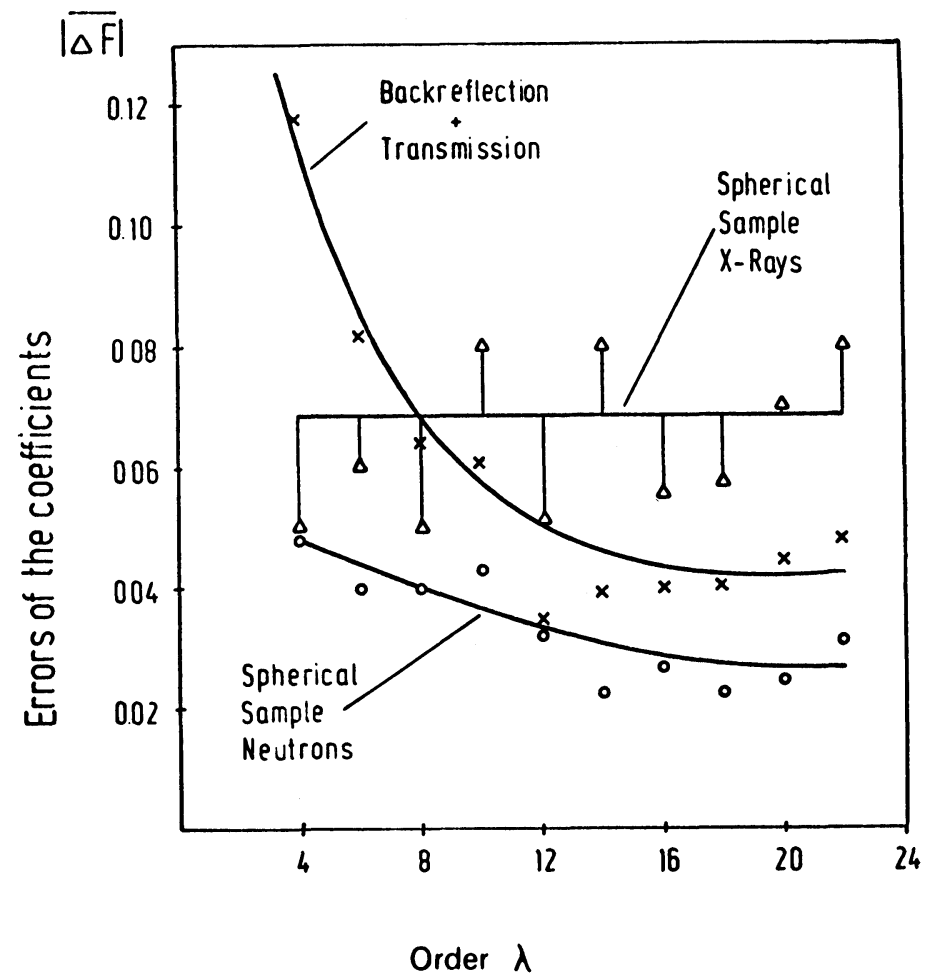

Figure 11 Error coefficients $\Delta F$ for pole figure measurements: $X$-ray transmissionreflection method; X-ray spherical sample method; neutron spherical sample method. 


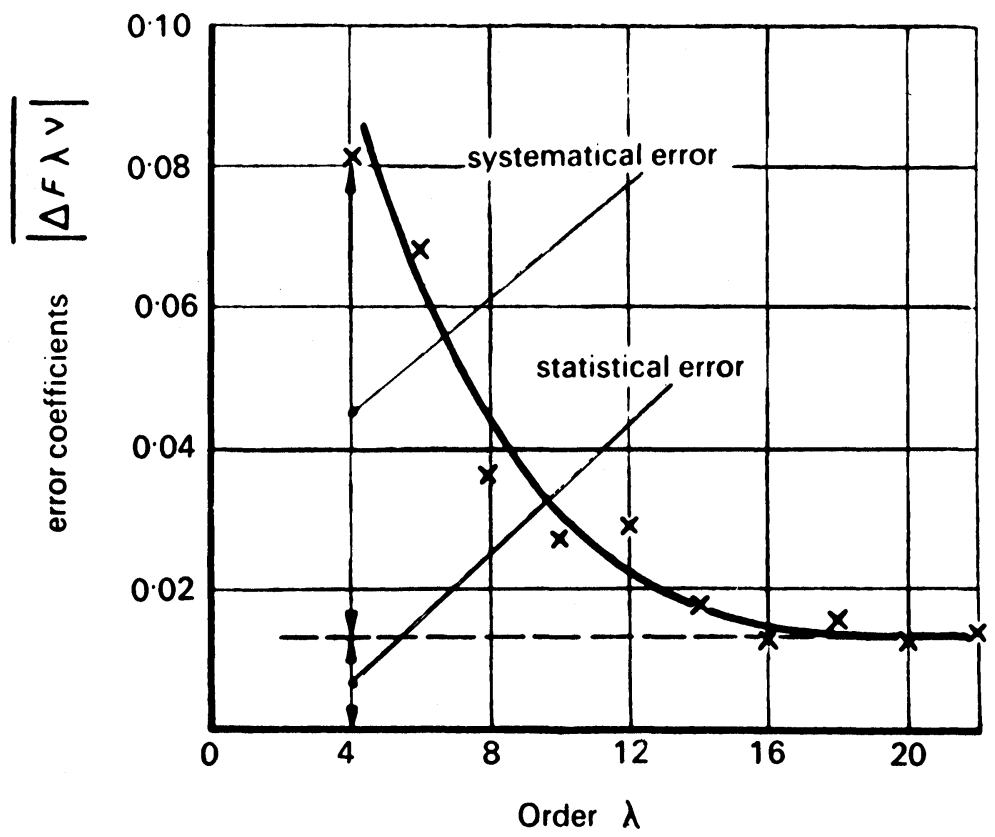

Figure 12 Statistical and systematical errors of pole figures expressed in the error coefficients $\left|\Delta F_{\lambda}^{v}\right|$.

which are particularly detrimental when ODF are to be calculated from pole figure measurements.

\section{MULTIPHASE MATERIALS}

A particularly strong influence of the absorption factor on X-ray texture measurements occurs in the case of multiphase materials, if the shape and arrangement of the phases is anisotropic. In Figure 13 the absorption factor of a directionally solidified $\mathrm{Pb}-\mathrm{Sn}$ eutectic is shown as a function of the angle $\gamma$ of the diffraction plane relative to the lamellae plane (Bunge, Liu, Hanneforth, 1987). It is seen that in this case the influence of absorption is so strong that texture measurements by X-ray diffraction become virtually impossible. 


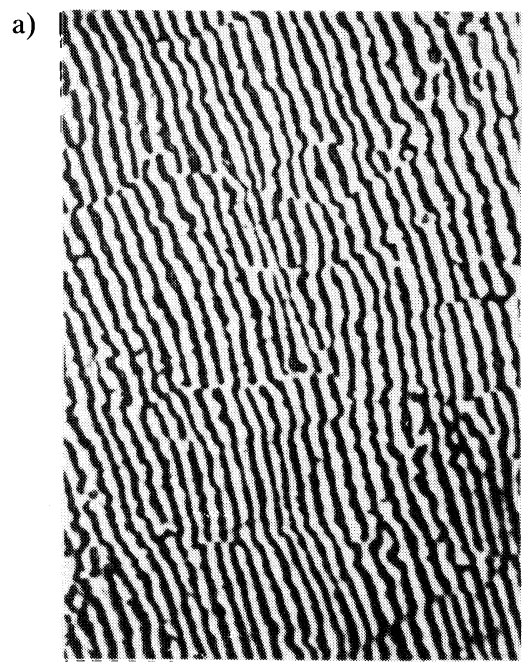

b)

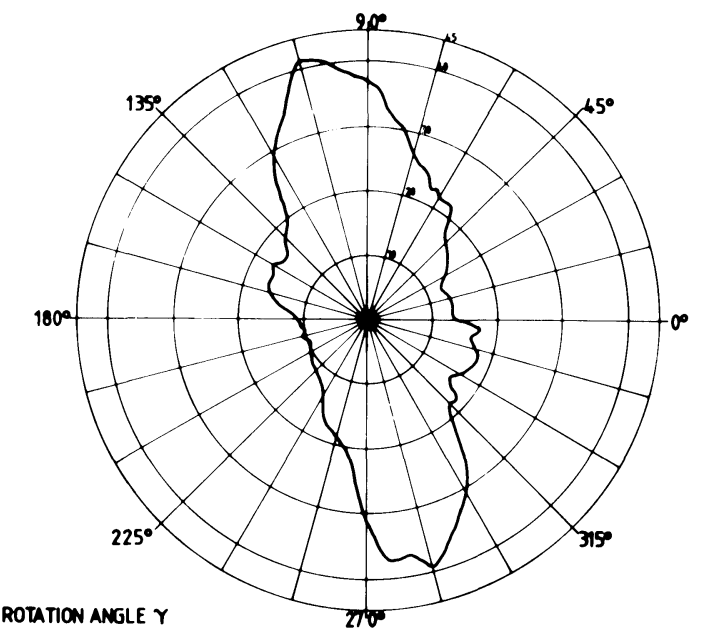

Figure 13 Anisotropic absorption of X-rays in lamellar structures. a) Metallographic section of a directionally solidified $\mathrm{Pb}-\mathrm{Sn}$ eutectic b) Absorption factor as a function of the angle $\gamma$ between lamellar plane and diffraction plane. 


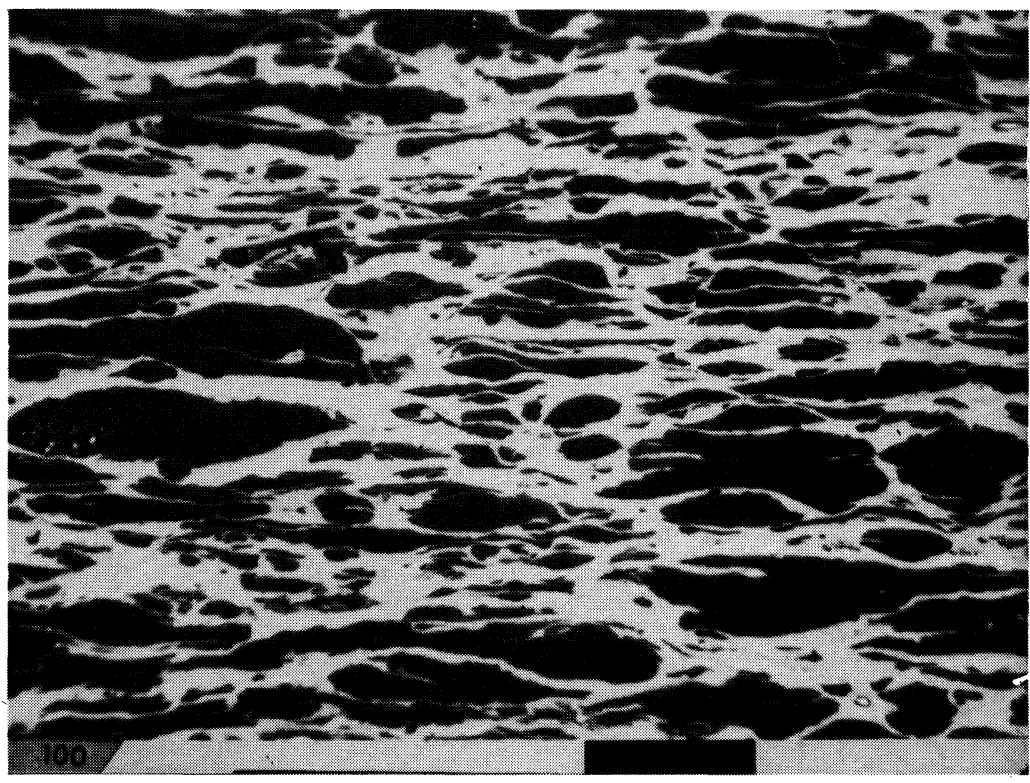

Figure 14 Metallographic section of a $90 \%$ extruded $\mathrm{Al}-\mathrm{Pb}$ composite.

The situation is similar in highly deformed two-phase materials e.g. 90\% extruded $\mathrm{Al}-\mathrm{Pb}$ composites as shown in Figure 14 (Brokmeier, Böcker and Bunge, 1988). Also in this case the X-ray absorption factor varies by as much as $25 \%$. Hence, X-ray texture measurements are falsified by this amount. In neutron diffraction this influence is in the range of $0.1 \%$. Figure 15 shows inverse pole figures of $\mathrm{Al}$ and $\mathrm{Pb}$ of a highly extruded $\mathrm{Al}-\mathrm{Pb}$ composite obtained by neutron diffraction. These measurements are free of errors due to anisotropic absorption.

\section{SMALL VOLUME FRACTIONS OF SECOND PHASES}

It is interesting to study the texture of a phase which is present in a multiphase material only in small volume fractions e.g. below $1 \%$. 

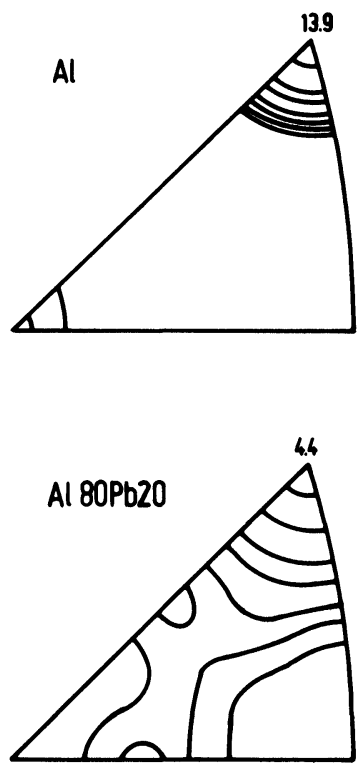
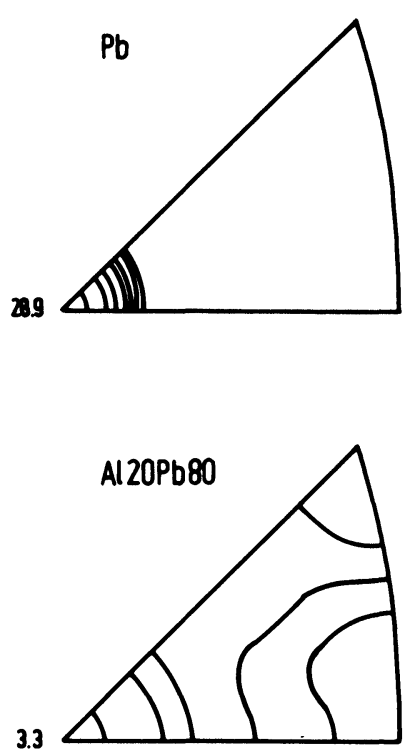

Figure 15 Inverse pole figures of the $\mathrm{Al}$ and $\mathrm{Pb}$-phase of pure metals and composites extruded $90 \%$ measured by neutron diffraction.

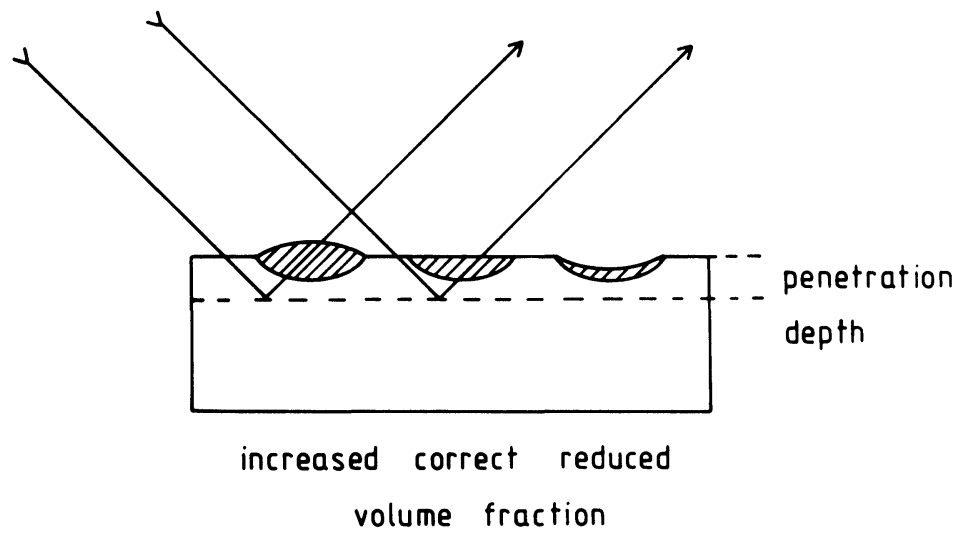

Figure 16 Possible influences of sample preparation on small particles of a second phase in the sample surface. 


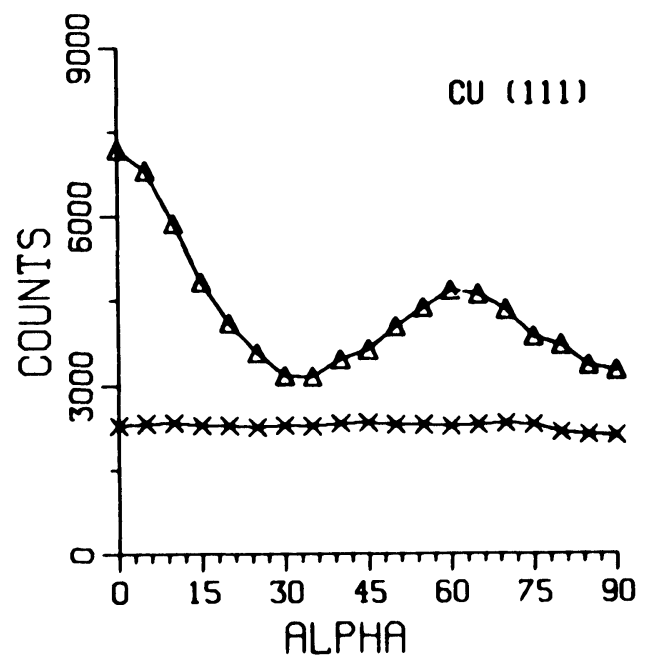

Figure 17 Pole figure of the copper phase of an extruded $\mathrm{Al}-\mathrm{Cu}$ composite with $1 \% \mathrm{Cu}$.

In this case, $\mathrm{X}$-ray texture analysis must assume that the area fraction of the phase in the investigated surface is the same as the volume fraction. With a small volume fraction this may not be the case simply because of poor statistics. Furthermore because of different properties of the phases, the surface of the particles may not be flat as is shown schematically in Figure 16. Hence, X-ray texture measurements in phases of less than about 2 or $3 \%$ become virtually impossible. In neutron diffraction, on the other hand, the whole sample volume contributes to the measurement and artefacts at the surface do not play any important role. Hence, neutron texture measurements can be carried out with reasonable accuracy well below 1 Vol.\%. Figure 17 shows for instance the pole figure of the copper phase of an $\mathrm{Al}-\mathrm{Cu}$ composite with $1 \% \mathrm{Cu}$. Recently measurement with $0.1 \%$ of copper have also been carried out.

\section{SAMPLE PROTECTION}

Another advantage of the high penetration depth of neutrons compared with X-rays is that it is easily possible to provide a 
neutron sample with a protective coating. This may be interesting when studying textures in oxygen-sensitive or hygroscopic materials. Similarly, different kinds of environments may be easily applied to a texture sample, e.g. high temperatures. In this case, the Eulerian cradle can be easily shielded from the heating device with shields the thicknesses of which are in the millimeter range.

\section{INFLUENCE OF THE ATOMIC NUMBER}

It was already mentioned, that the absorption coefficient of neutrons is smaller than that of X-rays by two or three orders of magnitude. This allows much bigger samples to be used. On the other hand, also the scattering factors are much smaller. Hence, it is not only possible but, in most cases, also necessary to use bigger samples.

Absorption coefficient and scattering factor are shown in Figure 18 and 19 respectively (see e.g. Bacon, 1975; Squires, 1978). They are not only smaller than those for X-rays. Also the variation of these quantities with atomic numbers is quite different in the two cases. Both quantities show a systematic, strong increase with atomic number for X-rays which is not the case for neutrons. This has some consequences for neutron texture analysis. In neutron diffraction, neighbouring elements may have quite different scattering factors. Hence it is easily possible to study ordering effects in solid solutions of such elements. As far as texture measurements are concerned this may be an important advantage when directional ordering in polycrystalline materials is to be studied, for instance in iron-nickel alloys. Directional ordering is strongly related to the texture of the material. The situation is also quite different for the light elements. Hence, texture studies of light element phases (especially in the presence of other phases containing heavy elements) will be much easier with neutrons than with X-rays.

As a consequence of different scattering factors of the atoms also the structure factors of a crystalline phase may be strongly different in X-ray and neutron diffraction. In texture analysis this may be important, for instance, if a particular structure factor is zero in one of the methods. The corresponding pole figure cannot be measured in this case. An example is the important (0001) pole figure of 


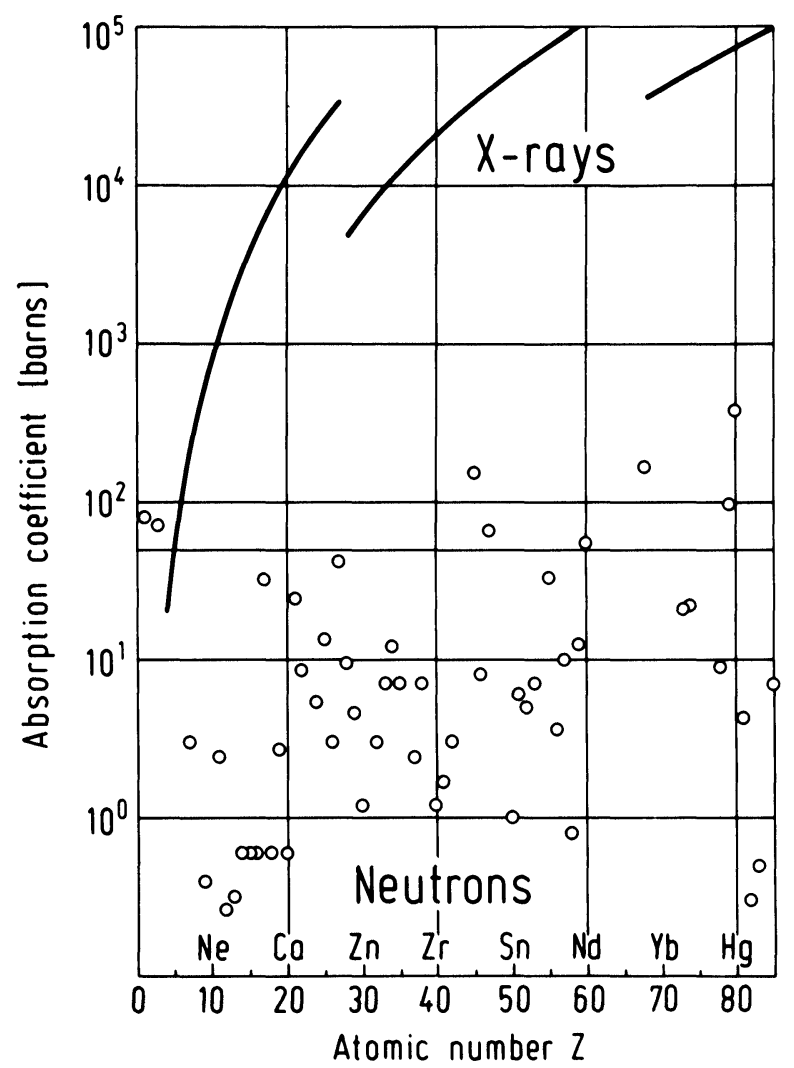

Figure 18 The absorption factor for $\mathrm{X}$-rays and neutrons as a function of atomic number.

$\mathrm{Al}_{2} \mathrm{O}_{3}$ ceramics. Its $\mathrm{X}$-ray intensity is lower than $1 \%$ of $(11 \overline{2} 3)$ which has the highest intensity. Hence, this pole figure cannot be measured by X-rays. The corresponding neutron diffraction intensity is, however, in the order of magnitude of about $10 \%$ of the maximum value. Hence it can well be measured by neutron diffraction. This is of great interest since $\mathrm{Al}_{2} \mathrm{O}_{3}$ substrates sometimes have a strong (0001) fibre texture which is well expressed in the (0001) pole figure. 


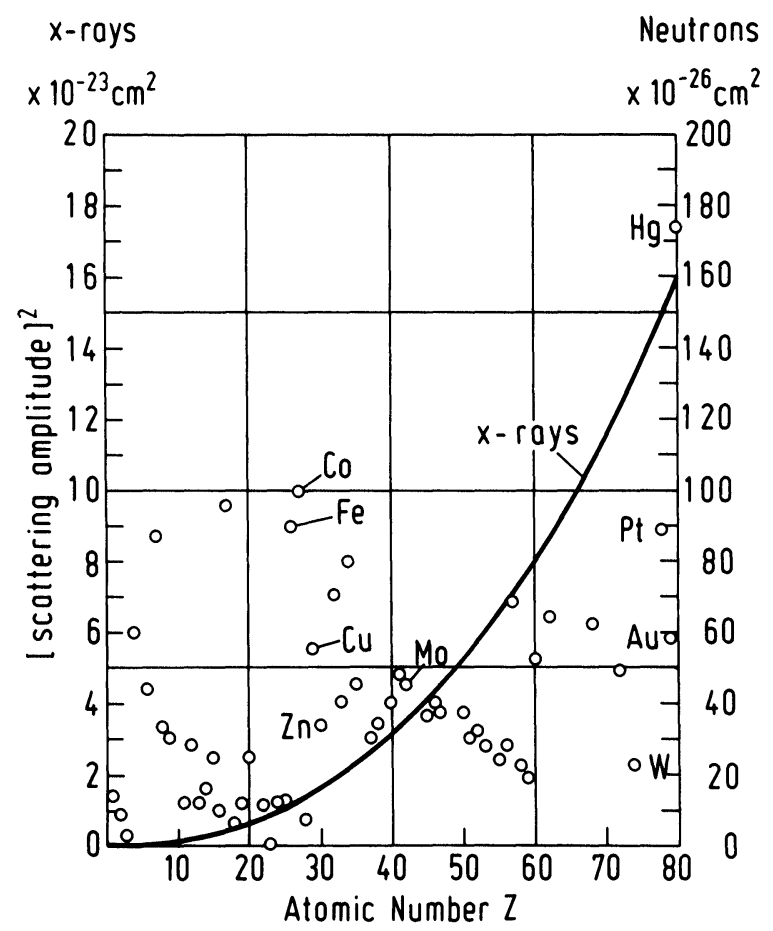

Figure 19 The scattering cross section of X-rays and neutrons as a function of the atomic number.

\section{INFLUENCE OF THE ISOTOPE}

The neutron scattering factors for various isotopes of the same element are usually quite different (till to opposite sign of the scattering factor). A particularly striking example of this effect is observed in hydrogen. Hydrogen itself has a low coherent scattering factor but shows strong incoherent scattering whereas the opposite is true for deuterium. As a consequence of this, neutron diffraction texture analysis in natural salt samples may be strongly complicated by the often observed presence of moisture in these materials. On the other hand, texture studies in ice, for instance, are facilitated if it is possible to use deuterated ice. 


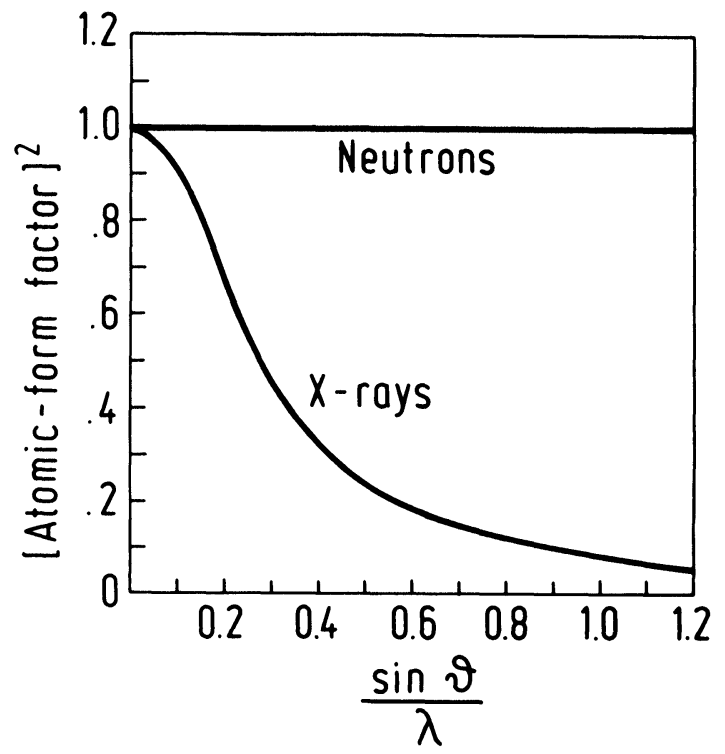

Figure 20 The scattering factor for X-rays and neutrons as a function of $\sin \vartheta / \lambda$.

\section{ANGULAR DEPENDENCE OF THE SCATTERING FACTOR}

X-ray scattering takes place in the whole volume of an atom whereas the most important part of neutron scattering comes from the nucleus the dimension of which is neglegible compared with the wavelength of the neutrons. Hence, the X-ray scattering depends on $\sin \vartheta / \lambda$ whereas neutron scattering is independent of this parameter, Figure 20. This difference is important for texture analysis especially when pole figures are to be converted into ODF. In this case the resolving power is the better the more pole figures can be used. Neutron diffraction then allows to measure also high index pole figures with a satisfactory accuracy. This is especially important for materials with low crystal symmetries.

\section{POSITION SENSITIVE DETECTORS}

In the conventional method of pole figure measurement the detector is placed at the Bragg angle $\vartheta$ with respect to the incident 
beam and an apperture is being used which allows the total (integrated) intensity of the diffraction peak to be measured. This way, one pole figure is obtained at a time. Then the position of the detector is changed to the next Bragg-position. Hence, the necessary pole figures are being measured sequentially. With the help of a position sensitive detector the whole $\vartheta$-spectrum can be measured at the same time. Figure 21 shows the instrument D1B at Grenoble, equipped with a position sensitive detector (Allemand et al., 1975). It is thus possible to measure all required pole figures simultaneously at the same time. This method provides two advantages:

1) the measuring time is greatly reduced

2) Overlapping pole figures can be separated

This latter point is especially important if materials with line-rich diffraction spectra are to be investiaged. Such spectra may be due to:

a) low symmetry

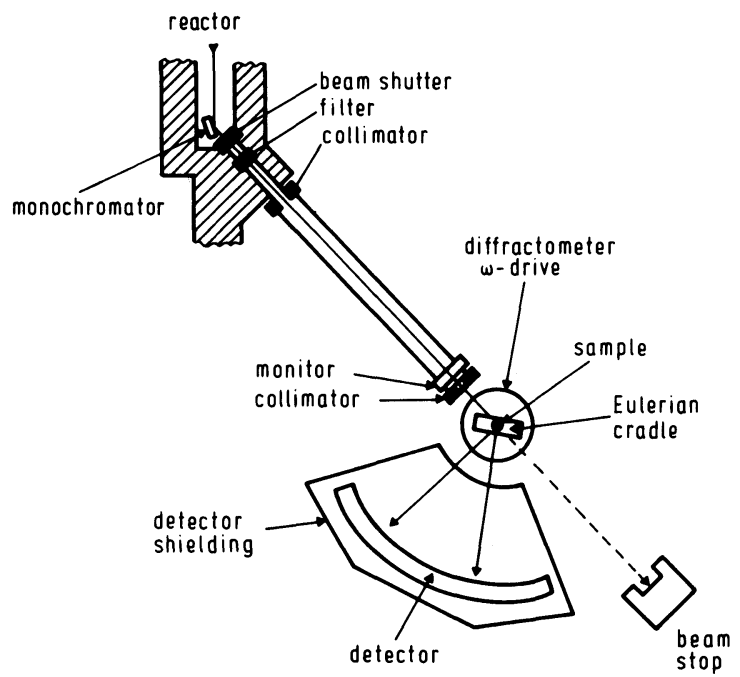

Figure 21 The instrument D1B at Grenoble used as a texture goniometer with position sensitive detector. 
b) high lattice constants

c) multiphase materials

Hence, this method is particularly suited to the study of:
a) intermetallic phases
b) ceramics
c) minerals
d) rocks
e) composites

Figure 22a shows for instance a felspare spectrum measured this way (Bunge, Wenk, Pannetier, 1982). An enlarged part of the spectrum is shown in Figure 22b. It is seen that line separation is possible by line profile analysis. It is also seen that a complete profile analysis improves the determination of background scattering and hence improves the accuracy of the measured integrated

a)

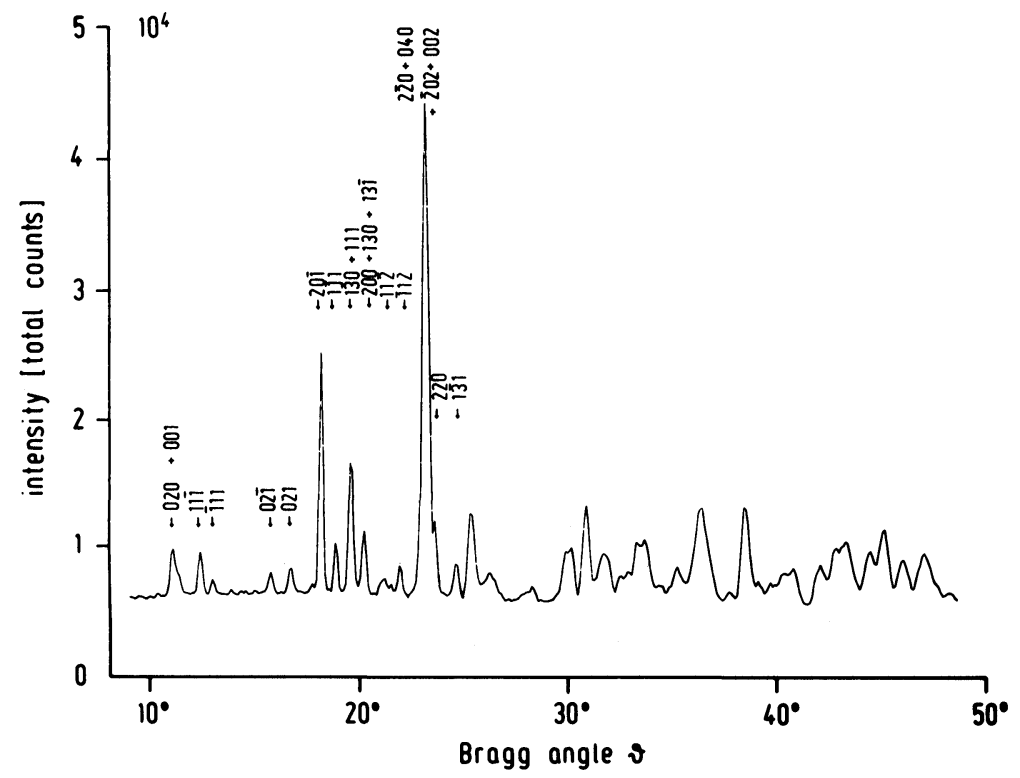

Figure 22 Diffraction spectrum of a felspare sample. a) the whole spectrum. 


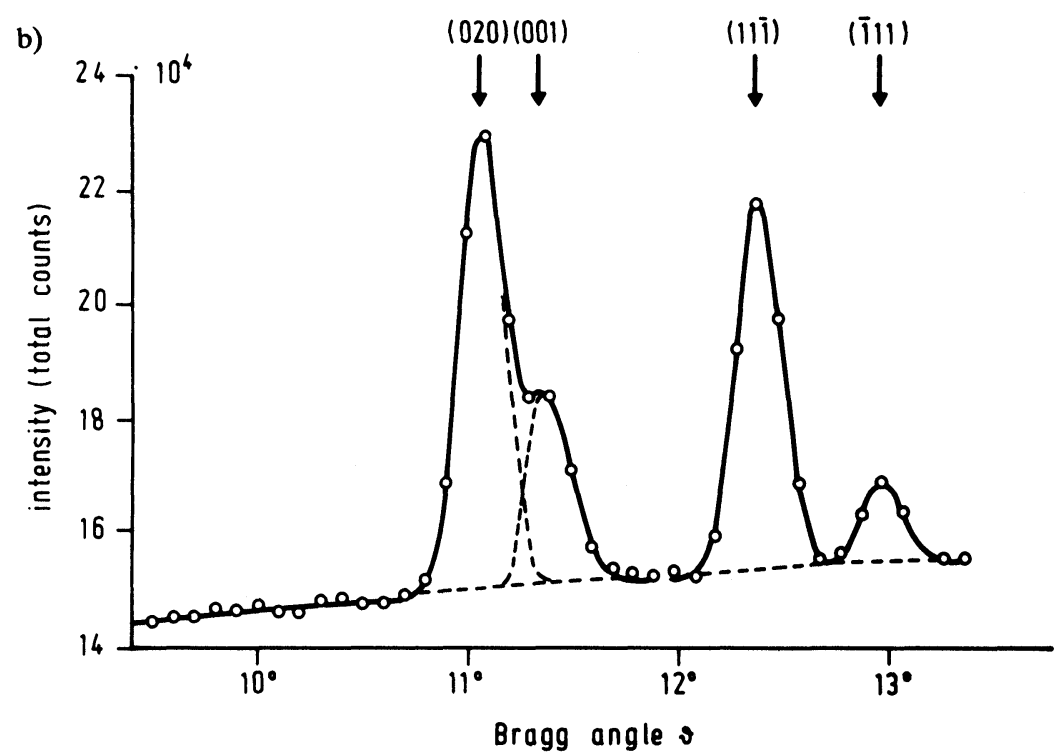

c)

$(1 \overline{3} 0)(130)$

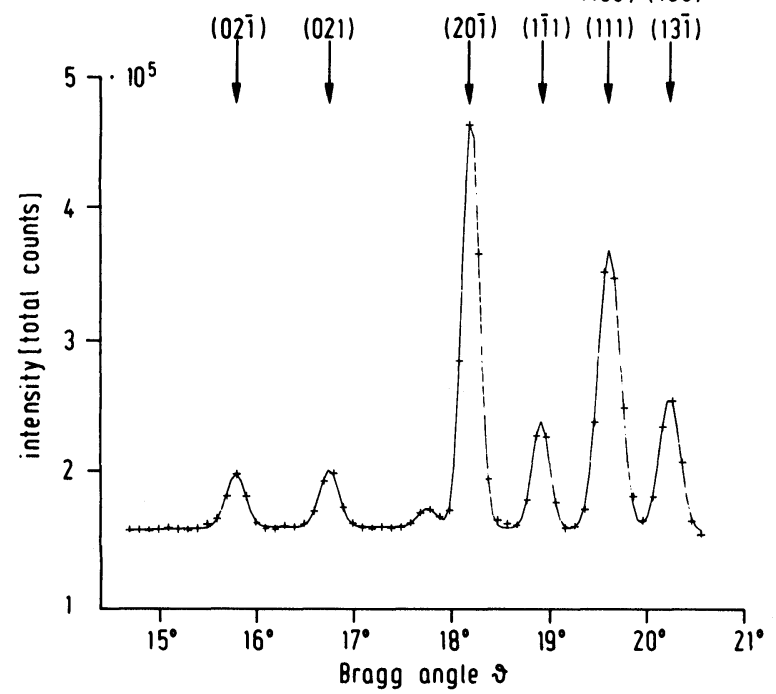

Figure 22 (Continued) b) partially overlapped peaks. c) totally overlapped peaks. 
intensity (Jansen, Schäfer and Will, 1986). Figure 22c finally shows another part of the spectrum. Here the overlapping of different diffraction peaks is total. These peaks cannot be separated "experimentally" by line profile analysis. They can however be separated "theoretically" in the process of ODF analysis using the special methods of "overlapping pole figures." This is possible for "intraphase" overlapping (Dahms and Bunge, 1987) as well as for "interphase" overlapping (Dahms, Brokmeier, Seute and Bunge, 1988).

When a position sensitive detector is being used the plane of the Eulerian cradle (plane of the $\chi$-rotation) can only be in "symmetry position" for one specific Bragg angle e.g. the angle $\vartheta_{m}$ in Figure 23. Only for this angle, the $\chi$-circle passes through north and south pole of the pole sphere. For all other $\vartheta$-angles $\chi$ and $\phi$ rotation gives a pole figure with a "blind area" in the vincinity of north- and south pole as is shown in Figure 24. Hence, in order to obtain complete pole figures an additional scan is necessary in order fill in the blind area and a coordinate transformation is necessary by which the angles $\omega \chi \phi$ of the Eulerian cradle are transformed into the pole figure angles $\alpha \beta$ (Bunge, Wenk, Pannetier, 1982). Some of

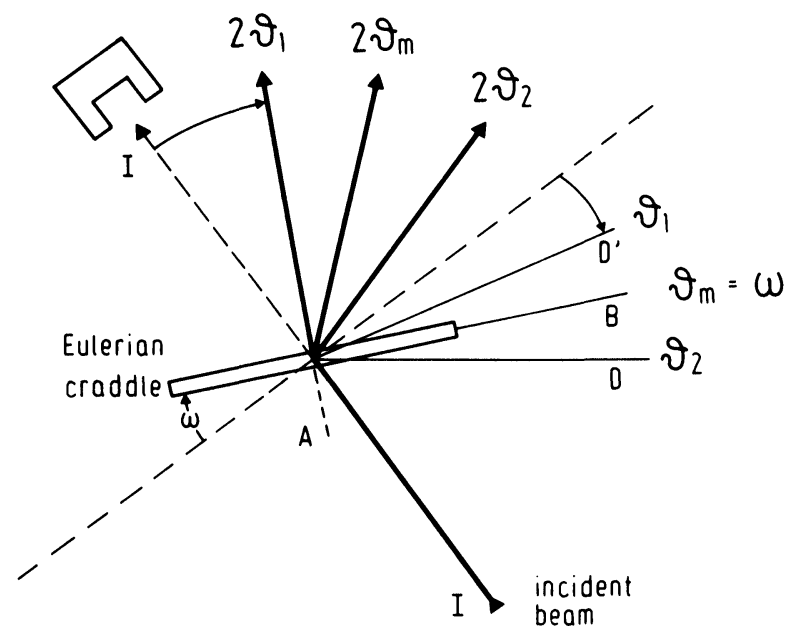

Figure 23 Adjustment of the Eulerian cradle in "symmetry position" for one of the Bragg angles $\vartheta_{m}$. 


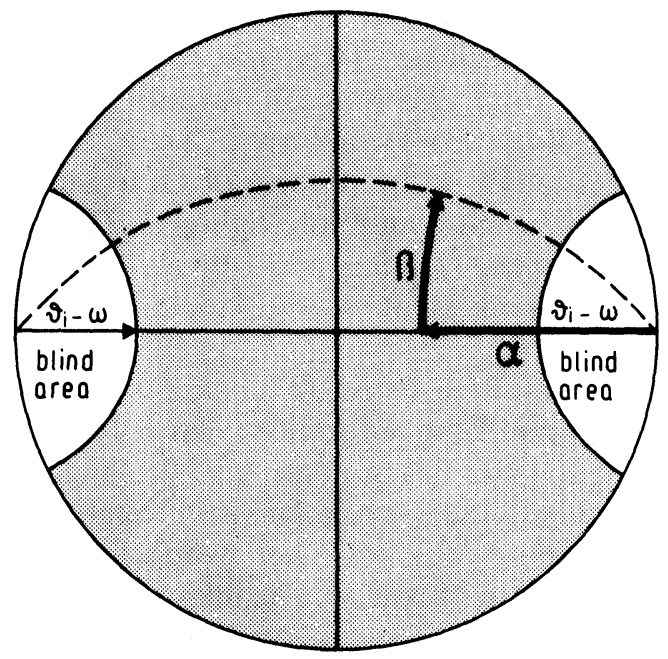

Figure 24 Pole figure scan for "off-symmetry" Bragg angles showing blind areas.
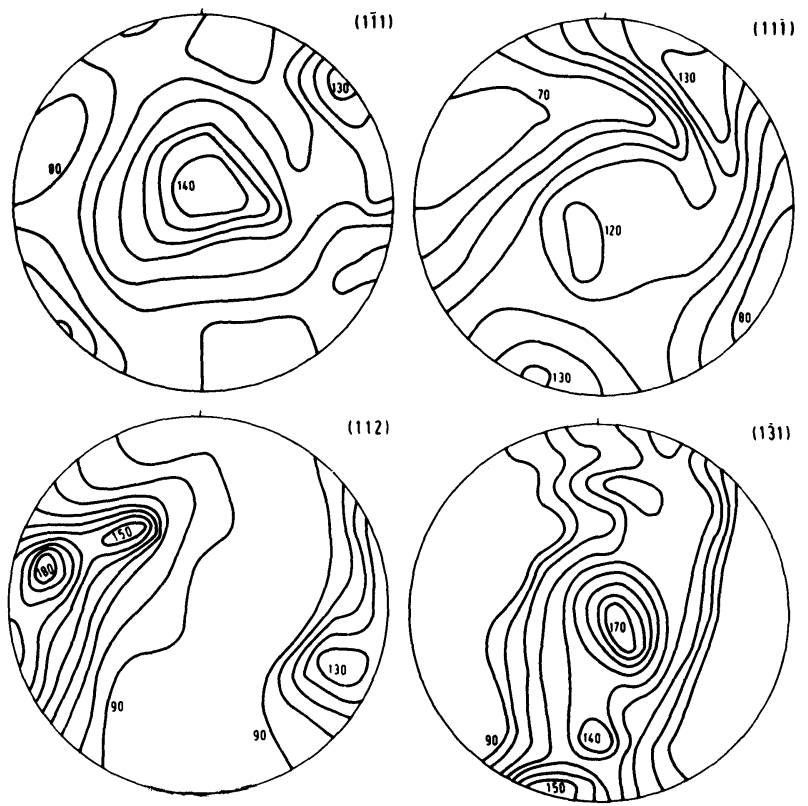

Figure 25 Four pole figures of a felspare sample. 
the felspare pole figures measured this way are shown in Figure 25 (Wenk, Bunge, Jansen and Pannetier, 1986).

In Figure 21 the position sensitive detector was used in "Bragg angle orientation ." In this orientation it measures one pole figure point of several Bragg angles at the same time. It can, however, also be used in "pole figure orientation" as is shown in Figure 26a (Juul-Jensen and Kjems, 1983). In this case the wavelength must be
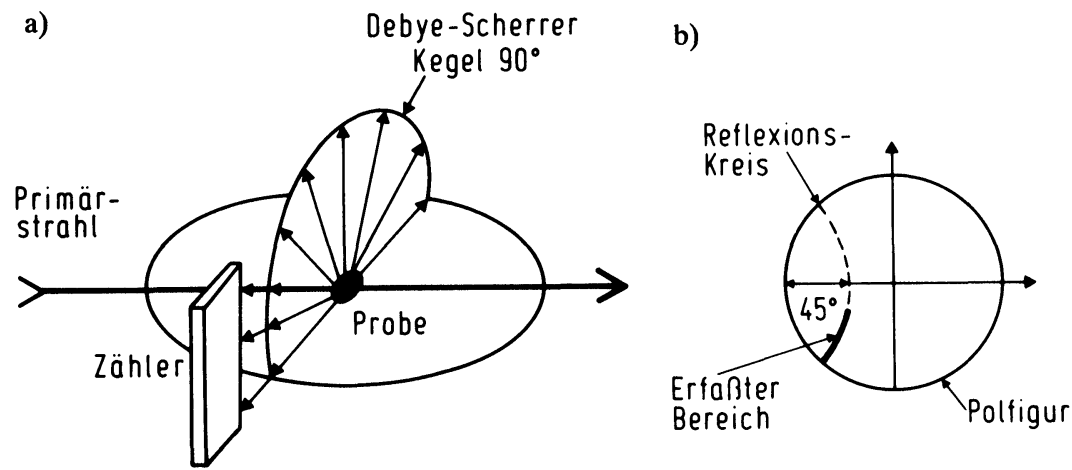

c)

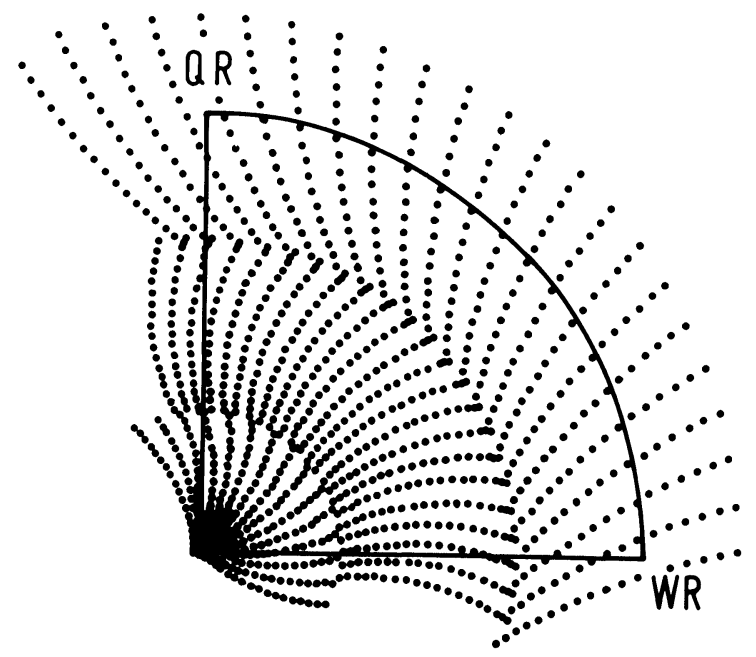

Figure 26 A position sensitive detector used in "pole figure orientation". a) Positioning of the detector at $2 \vartheta=90^{\circ}$. b) Scanned line in the pole figure. c) Complete pole figure scan. 
chosen such that $2 \vartheta=90^{\circ}$. The detector then measures a whole line of pole figure points but only in one pole figure as is shown in Figure 26b. In order to scan a whole pole figure completely a greatly reduced number of steps is then sufficient, as is shown in Figure 26d. This method has especially been applied for rapid pole figure measurment, e.g. real-time recystallization and phase transformation stuides (e.g. Juul-Jensen, 1986).

\section{WAVE LENGTH DISPERSIVE METHOD}

Beside the normal angular dispersive measurement also a wave length dispersive method can be used. In this case, white radiation is being used. The reflection at different lattice planes is then obtained with different wavelengths but at the same Bragg angle

$$
n \cdot \lambda_{(h k l)}=2 d_{(h k l)} \cdot \sin \vartheta
$$

The wavelength of neutrons is correlated with their velocity

$$
\lambda=\frac{h}{m \cdot v}
$$

Hence, wavelength measurement can be obtained by time-of-flight measurement and this, in turn, requires to fix the starting time of a neutron. Time of flight measurement requires narrow pulses of neutrons following each other in rather long time intervals. A pulsed beam can be obtained mainly in two ways:

a) a continuous beam can be "chopped" into short pulses using a chopper

b) The neutrons can be directly produced in pulses e.g. in a pulse reactor or by a pulsed spallation source.

It is thus necessary to measure the time of each incoming neutron registered in the detector. The "time spectrum" obtained with one neutron pulse thus corresponds with the wavelength spectrum and hence with the $d$-value spectrum. Such spectra are then to be added up in a multi-channel analyzer for a sufficient number of neutron pulses. Finally a time-of-flight spectrum is obtained as shown for instance in Figure 27 (Feldmann, 1986). Such spectra have to be 


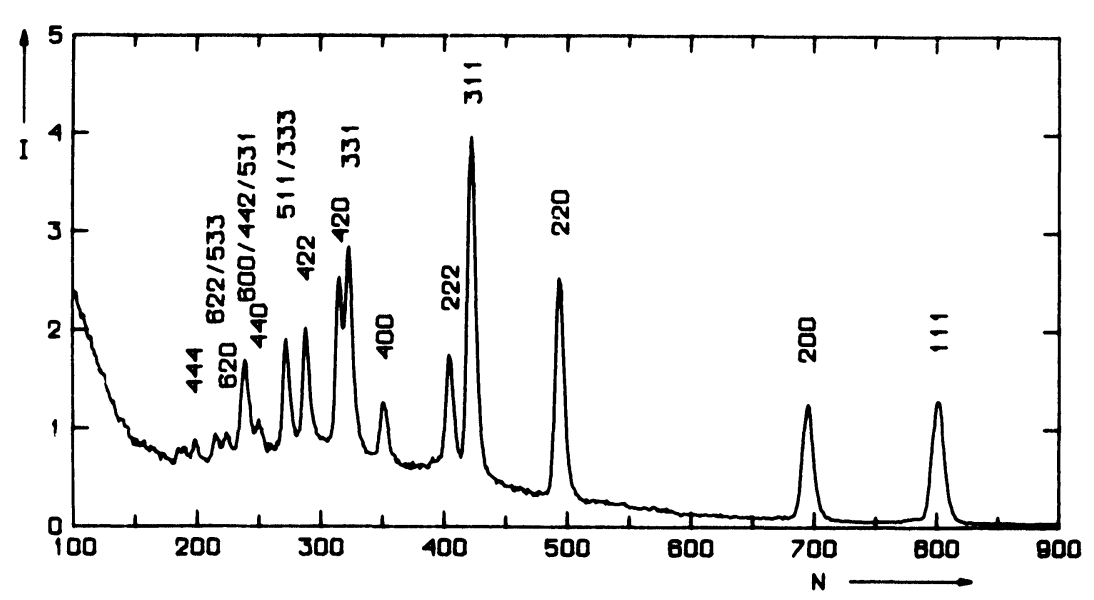

Figure 27 Time-of-Flight spectrum of copper.

measured for all necessary sample orientations in order to obtain all pole figures at the same time. Thus far, this method is similar to the method using a position sensitive detector. In contrast to the latter one, however, all pole figures are measured with the same Bragg-angle, i.e. in "symmetry position" as in the sequential angular dispersive method. Hence, a blind area does not occur in this method and no transformation from $\omega \chi \phi$ to $\alpha \beta$ is necessary.

\section{COMBINATION OF PSD AND TOF METHOD}

The three methods mentioned above may be combined into one method i.e.

a) PSD in Bragg-angle direction

b) PSD in pole figure direction

c) TOF method

This requires a pulsed neutron source and a two-dimensional position sensitive detector which is at the same time also timesensitive. This method shown in Figure 28 has been used by Vergamini and Wenk (1988). It gives-at the same time-a 


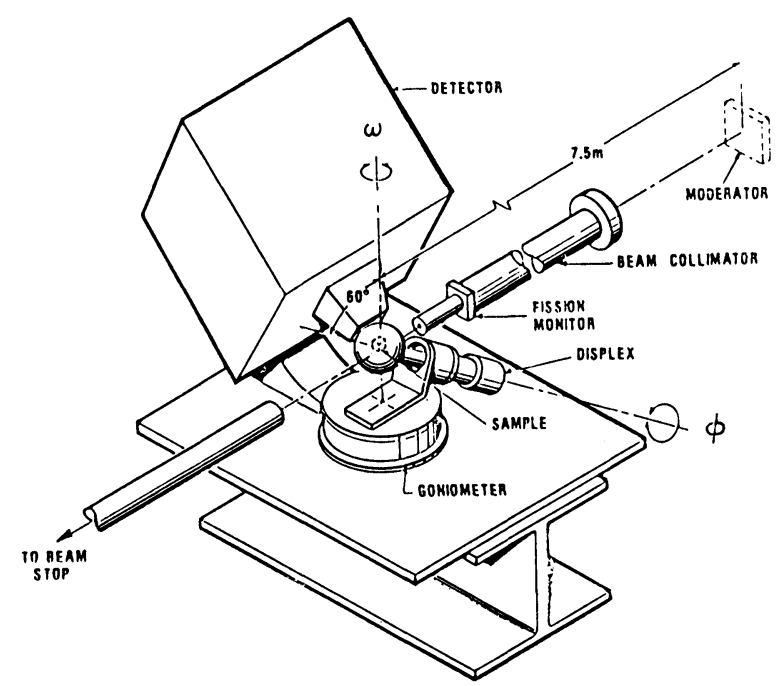

Figure 28 Texture measurement with a two-dimensional TOF-method.

two-dimensional area of a large number of pole figures. Hence, this method is the most economic one.

\section{MAGNETIC TEXTURE ANALYSIS}

A second part of neutron scattering is due to magnetic interaction of the neutron's magnetic dipole moment with the distribution of the magnetic moment of the electrons in the atom. Hence, this part of neutron scattering depends on $\sin \vartheta / \lambda$ in a similar way as X-ray scattering, Figure 29 (see e.g. Bacon, 1975). In the case of non-polarized neutrons the magnetic part of the reflected intensity is proportional to

$$
q^{2} \sin ^{2} \alpha
$$

where $\alpha$ is the angle between magnetization direction and the diffraction vector $s$ i.e. the normal direction to the reflecting lattice plane as is seen in Figure 30. Magnetic neutron scattering is related to the magnetic crystal structure of the material which may be different from the "chemical" crystal structure. The most important 


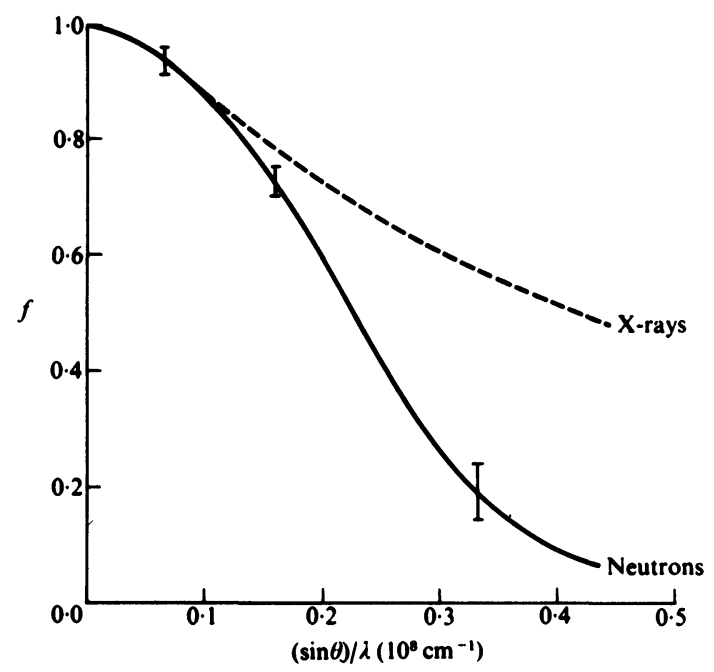

Figure 29 The magnetic scattering factor of neutrons depends on $\sin \vartheta / \lambda$.

cases of magnetic structures are the ferromagnetic and antiferromagnetic case. In the first case all magnetic moments of the atoms are parallel to a certain crystal direction $m$, in the second case they are mutually antiparallel. This means that in the first case the unit cell is the same as the chemical unit cell but crystal symmetry is different and in the second case unit cell and symmetry both are changed. From the view point of texture analysis the ferromagnetic case is most important. With an applied magnetic
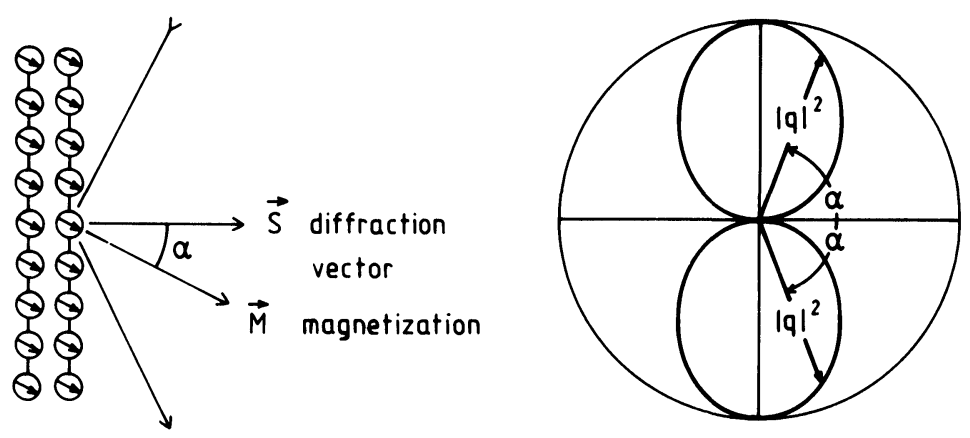

Figure 30 The magnetic scattering of unpolarized neutrons depends on the angle $\alpha$ between magnetization direction and normal direction to the reflecting lattice plane. 
field $H$ the magnetization direction $m$ may be parallel to any crystal direction $h$. Two limiting cases may be particularly considered. In the case of zero magnetic field the magnetization direction is parallel to one of several crystallographically equivalent directions $h_{0}^{i}$. In the case of saturation it is parallel to the field direction

$$
\begin{array}{ll}
m \| h_{0}^{i} & H=0 \\
m \| H & H \rightarrow \infty
\end{array}
$$

In the second case the magnetization direction is uniquely fixed in each crystal. It is parallel to the same sample direction but to different crystal directions which are however uniquely determined by crystal orientation $g$. In the first case the distribution of magnetization directions over the crystal directions $h_{0}^{i}$ will generally depend on the magnetic history of the material. Different directions $h_{0}^{i}$ may occur in one and the same crystallite which is then divided into magnetic domains. In this case the "magnetic texture" can be easily defined as the orientation distribution function of the domains. We specify a crystal coordinate sytem $K_{B}^{M}$, the $x_{3}$-axis of which is parallel to the magnetization direction $h_{0}^{i}$ of the considered domaine. The orientation of this coordinate system with respect to the sample coordinate system $K_{A}$ is described by the rotation $g^{M}$. The magnetic texture is then the volume fraction of domains having an orientation $g^{M}$ within the angular range $d g^{M}$

$$
f^{M}\left(g^{M}\right)=\frac{d V\left(g^{m}\right) / V}{d g^{M}}
$$

This definition of the magnetic texture is completely analogous to Eq. (1), only the symmetry of the magnetic texture function is different from that of the crystallographic texture. As an example, in cubic ferromagnets the magnetization direction is often parallel to the cubic axes

$$
h_{0}^{i}=\langle 100\rangle
$$

The magnetic crystal symmetry is then tetragonal instead of cubic. Also the sample symmetry may be different from the conventional sample symmetry due to the distribution of magnetization directions over the various $h_{0}^{i}$-directions. Also the pole figures of this magnetic texture are defined in the same way as in the crystallographic 
texture (see e.g. Mücklich, Hennig, Bouillot and Matthies, 1984)

$$
P_{h}^{M}(y)=\frac{d V / V}{d y}
$$

with the only difference that now $h$ is the normal direction to the reflecting lattice plane $(h k l)$ indexed in the magnetic coordinate system $K_{B}^{M}$ i.e. with the lower "magnetic" crystal symmetry (e.g. tetragonal). The pole figure $P_{h}^{M}(y)$ can, however, not be measured directly by neutron diffraction. Rather all those pole figures are systematically superposed which belong to crystallographically equivalent directions $h^{i}$.

$$
P_{\{h\}}^{M}(y)=\sum_{i=1}^{I_{n}} q_{i}^{2} \cdot P_{h^{i}}^{M}(y)
$$

The superposition factors $q_{i}^{2}$ are known according to Eq. (9). Pole figure inversion, i.e. the calculation of the ODF $f^{M}(g)$ from pole figures $P_{h}^{M}(y)$ can be carried out with superposed pole figures $P_{\{h\}}^{M}(y)$ instead of the no-superposed ones (see e.g. Dahms and Bunge 1987). Hence, information about the magnetic texture can be obtained from the magnetic part of neutron diffraction pole figures (see also Bunge (in print), 1989).

\section{CONCLUSIONS}

Texture measurements can be carried ou by neutron diffraction much in the same way as by X-ray diffraction, i.e. by pole figure measurement followed by pole figure inversion. Because of the specific properties of the two radiations there are, however, some differences between the two method as far as the quantitative relations are considered. These differences are mainly in favour of neutron diffraction, whereas the main drawback of this radiation (compared to X-rays) is its limited availability and high cost of neutron diffraction equipment and especially of the neutron source. The advantages of neutron diffraction are, first of all, due to the lower absorption coefficient. As a consequence of this, much bigger samples can be used. 
- This in turn leads to a much better grain statistics. Hence, samples with one order of magnitude bigger grain sizes can be succesfully studied by neutrons.

- The remaining absorption correction is usually much smaller with neutrons and can be calculated with a much higer precission. Hence, the experimental errors of neutron diffraction pole figures may be an order of magnitude lower than those of X-ray diffraction. This is particularly valuable for high precision ODF calculations.

- The lower error allows texture analyses of second phases which are present with only very low volume fractions.

- The high penetration depth allows the global texture of technologically interesting work pieces to be measured directly.

-There is virtually no anisotropic absorption effect in anisotropic polyphase materials as is the case in X-ray diffraction.

-A further advantage of neutron diffraction is due to a scattering factor independent of $\sin \vartheta / \lambda$. This allows high-index pole figures to be easily measured.

Neutron diffractometers are often equipped with position sensitive detectors which have not yet become standard equipment in X-ray diffraction. A PSD in $\vartheta$-direction allows the measurement of many pole figures at the same time. Furthermore, it allows the seperation of overlapping peaks which is particularly valuable for materials with big unit cells and low symmetries as well as multiphase materias. Finally, neutron scattering contains a magnetic part which allows magnetic textures to be measured which is by no means possible using $\mathrm{X}$-ray diffraction.

\section{References}

Allemand, R., Bourdel, J., Roudant, F., Couvert, P., Ibel, K., Jacobe, J., Cotton, J. P. and Faroux, B. (1975). Position-sensitive Detectors (PSD) for Neutron Diffraction. Nucl. Instr. Methods 126, 29-42.

Bacon, G. E. (1975). Neutron Diffraction. The Clarendon Press Oxford.

Bankwitz, P., Betzl, M., Drechsler, L. P., Feldmann, K., Fuentes, L., Kaempf, H., Matthies, S. and Walter, K. (1984). Investigation of Preferred Orientations of the Quartz Crystallites in Granulite Rock by Neutron Time-of-Flight Diffraction. Proc. ICOTOM 7 Noordwijkerhout 473-478.

Betzl, M., Drechsler, L. P., Feldmann, K., Fuentes, L., Hennig, K., Kleinstück, K. H., Matthies, S., Matz, W., Tobisch, J. and Walther, K. (1984). Progress in 
Neutron Time-of-Flight Texture Studies at Pulsed Reactor IBR-30. Proc. ICOTOM 7 Noordwijkerhout 753-758.

Brockhouse, B. N. (1953). The Initial Magnetization of Nickel Under Tension. Canad. J. Phys. 331, 353-355,

Brokmeier, H. J. (1982). Texturuntersuchungen an natürlichen und experimentell verfestigten Halit-Gesteinen mit Neutronen und W Strahlung. Thesis Clausthal.

Brokmeier, H. G., Böcker, W. and Bunge, H. J. (1988). Neutron Diffraction Texture Analysis in Extruded Al-Pb Composites. Textures and Microstructures 8 \& 9, 429-441.

Bunge, H. J. (1966). Ein Zuverlässigkeitskriterium für Polfiguren von Texturen kubischer Metalle. Kristall u. Technik 1, 171-173.

Bunge, H. J. (1969). Mathematische Methoden der Texturanalyse. Akademie-Verlag Berlin.

Bunge, H. J. (1982). Texture Analysis in Materials Science. Butterworths Pub. London.

Bunge, H. J. (1987). Three-dimensional Texture Analysis. International Materials Review 32, 265-291.

Bunge, H. J. (1989). Texture and Magnetic Properties. Textures and Microstructures (in print).

Bunge, H. J., Liu, Y. S., Hanneforth, R. (1987). Anisotropic Absorption of X-rays in Polyphase Materials. Scripta Met. 21, 1423-1427.

Bunge, H. J. and Puch, K. H. (1984). Principles of Texture Goniometer Measurement. Z. Metallkde. 75, 124-132.

Bunge, H. J., Schleusner, D. and Schläfer, D. (1974). Neutron Diffraction Studies of the Recrystallization Textures in Cold Rolled Low-Carbon Steel. Metal Science Journal 8, 413-423.

Bunge, H. J. and Tobisch, J. (1972). The Texture Transition in $\alpha$-Brasses Determined by Neutron Diffraction. J. Appl. Cryst. 5, 27-40.

Bunge, H. J., Tobisch, J. and Sonntag, W. (1971). On the Development of the Rolling Texture in Copper Measured by Neutron Diffraction. J. Appl. Cryst. 4, 303-310.

Bunge, H. J. and Tobisch, J. (1968). Bestimmung der Walztextur des Kupfers mit Hilfe der Neutronenbeugung. Z.f. Metallkunde 59, 471-475.

Bunge, H. J., Tobisch, J. and Mücklich, A. (1974). The Development of Rolling Texture in $\alpha$-Brass Determined by Neutron Diffraction. Texture 1, 211-231.

Bunge, H. J., Wenk, R. and Pannetier, J. (1982). Neutron Diffraction Texture

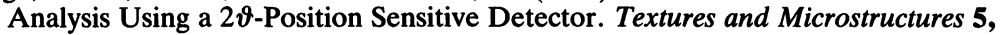
153-170.

Dahms, M., Brokmeier, H. G., Seute, H. and Bunge, H. J. (1988). Quantitative Texture Analysis in Multiphase Materials with Overlapping Bragg Reflections. Proc. of the 9th International Conference Risø.

Dahms, M. and Bunge, H. J. (1987). Texture Analysis Using Totally and Partially Coincident Pole Figures. In: Theroretical Methods of Texture Analysis. Ed. H. J. Bunge. DGM Informationsgesellschaft Oberursel 143-158.

Drechsler, L. P., Feldmann, K., Frischbutter, K. and Walter, K. (1988). Neutronographic Fabric Analysis of Quartz in Naturally Deformed Gneiss. Textures and Microstructures 8 \& 9, 737-750.

Feldmann, K. (1986). Application of Neutron Time of Flight Diffraction to Texture Studies. In: Experimental Techniques of Texture Analysis. Ed. H. J. Bunge. DGM Informationsgesellschaft Oberursel 253-264.

Feldmann, K. (this volume). Texture Investigations by Neutron Time-of-Flight Diffraction. Textures and Microstructures 12, 309-323. 
Feldmann, K., Andreeff, A., Betzl, M., Hennig, K., Kleinstück, K., Matz, W. and Walther, K. (1981). Quantitaive Texture Analysis by Neutron Time-of-Flight Method. Proc. ICOTOM 6 Tokyo 1192-1201.

Feldmann, K., Betzl, M., Andreeff, A. Henning, K., Kleinstück, K. and Matz, W. (1980). Comparison of Quantitative Textures Analysis Results From Time-of Flight and Conventional Neutron Diffraction. Texture of Crystalline Solids 4, $1-12$.

Hennig, K., Mücklich, A., Novikov, I. I., Portnoy, V. K., Ilenko, V. M. and Matthies, D. (1984). Study of Superplasticity of $\mathrm{Al}-5 \mathrm{Ca}-5 \mathrm{Zn}$ via Neutron Diffraction Texture Analysis. Proc. ICOTOM 7 Noordwijkerhout 499-502.

Hennig, K., Wieser, E., Betzl, M., Feldmann, K. and Mücklich, A. (1981). Magnetic Texture (Magnetic Pole Figures) Proc. ICOTOM 6 Tokyo 967-974.

Höfler, S., Schaefer, W. and Will, G. (1986). Texture Measurements at the Neutron Diffractometer in Jülich. In: Experimental Techniques of Texture Analysis. Ed. H. J. Bunge. DGM Informationsgesellschaft Oberursel 241-252.

Jansen, E., Schaefer, W. and Will, G. (1986). Application of Profile Analysis Methods in Texture Measurements Using Position-Sensitive Detectors. In: Experimental Techniques of Texture Analysis. Ed. H. J. Bunge. DGM Informationsgesellschaft Oberursel 229-240.

Jensen, D., Hansen, N., Kjems, J. and Leffers, T. (1981). Neutron Diffraction Texture Measurement as a Tool For the Investigation of Recrystallization Kinetics. Proc. ICOTOM 6 Tokyo 1179-1191.

Juul-Jensen, D. and Kjems, J. K. (1983). Apparatus for Dynamical Texture Measurement by Neutron Diffraction Using a Position Sensitive Detector. Textures and Microstructures 5, 239-252.

Juul-Jensen, D. (1986). Fast Texture Measurement by Neutron Diffraction Using a Linear Position Sensitive Detector. In: Experimental Techniques of Texture Analysis. Ed. H. J. Bunge. DGM Informationsgesellschaft Oberursel 217-228.

Klimanek, P., Hensger, K. E., Kleinstück, K., Mücklich, A., Hennig, K. (1981). Neutron Diffraction Studies of Textures due to High-Temperature Thermomechanical Treatment (HTMT) of Steel. Proc. ICOTOM 6 Tokyo 680-689.

Klimanek, P., Mücklich, A. and Hennig, K. (1981) Neutron Diffraction Studies of Texture Changes Due to Cold Rolling of Two-phase Stainless Steel X5CrNiTi26.6. Proc. ICOTOM 6 Tokyo 901-909.

Kleinstück, K., Tobisch, J., Betzl, M., Mücklich, A., Schläfer, D. and Schläfer, U. (1976). Texturuntersuchungen von Metallen mittels Neutronenbeugung. Kristall u. Technik 11, 409-429.

Mücklich, A., Hennig, K., Bouillot, J. and Matthies, S. (1984). Magnetic Texture Study of an Fe-Si Base alloy by Means of Mösbauer Spectroscopy and Neutron Diffraction. Proc. ICOTOM 7 Noordwijkerhout 657-662.

Nosik, Yu. S., Oserow, R. P. and Hennig, K. (1979). Structural Neutronography. Atomisdad Moscow (russ.).

Puch, K. H., Klein, H. and Bunge, H. J. (1984). A New Computer Operated Texture Goniometer. Z. Metallkunde 75, 133-139.

Schäfer, W., Höfler, S. and Will, G. (1988). Application of Neutron Diffraction Pole Figure Measurements of Polycrystalline and Monocrystalline Metallic Samples. Textures and Microstructures 8 \& 9, 457-466.

Schläfer, D. (1968). Die Anwendung des Zuverlässigkeitskriteriums für Polfiguren kubischer Metalle. Kristall u. Technik 3, 473-476.

Schläfer, D. and Bunge, H. J. (1974). The Development of the Rolling Texture of Iron Determined by Neutron Diffraction. Texture 1, 157-171. 
Schreiber, U., Hötzsch, K., Kleinstück, K., Klimanek, P., Mücklich, A., Tobisch, J. and Hennig, K. (1978). Neutron Diffraction Analysis of Textures and its Development in Steels with a Micro Duplex Structure. Textures of Materials. Ed. G. Gottstein and K. Lücke Springer Verlag Berlin 317-324.

Schwarzer, R. and Weiland, H. (1986). Electron Diffraction Pole Figure Measurements. In: Experimental Techniques of Texture Analysis. Ed. H. J. Bunge. DGM Informationsgesellschaft, 287-300.

Squires, G. L. (1978). Thermal Neutron Scattering. Cambridge University Press.

Stott, D. E. and Hutchinson, W. B. (1973). Application of Neutron Diffraction to the Study of Texture and Magnetic Properties in Grain Oriented Silicon Iron. Proc. 3-colloque européen sur les textures de déformation et de recrystallization des métaux et leur application industrielle. Pont-à-Mousson 427-438.

Szpunar, J. (1976). Texture and Neutron Diffraction. Atomic Energy Rev. 14, 199-261.

Szpunar, J., Oles, A., Buras, B., Sosnowskaja, I. and Pietras, E. (1968). Texture Studies of Coarse-grained Metal Sheets by Means of Neutron Diffraction. Nucleonika 13, 1111-1118.

Tobisch, J. and Bunge, H. J. (1972). The Spherical Samples Method in Neutron Diffraction Texture Determination. Texture 1, 125-127.

Walther, K., Hinz, D. and Hennig, K. (1981). Texture Investigations into Pressed and Sintered $\mathrm{ZnO}$ Powder by Neutron Diffraction. Proc. ICOTOM 6 Tokyo 1049-1056.

Welch, P. I. (1986). Neutron Diffraction Texture Analysis. In: Experimental Techniques of Texture Analysis. Ed. H. J. Bunge. DGM Informationsgesellschaft Oberursel 183-208.

Wenk, H. R., Bunge, H. J., Jansen, E. and Pannetier, J. (1986). Preferred Orientation of Plagioclase, Neutron Diffraction and U-Stage Data. Tectonophysics 126, 271-284.

Wenk, H. R., Vergamini, P. J. and Larson, A. C. (1988). Texture Analysis by TOF Measurements of Spallation Neutrons With a 2D-Position Sensitive Detector. Textures and Microstructures 8 \& 9, 443-456. 